\title{
NUMERICAL APPROXIMATIONS FOR THE MOLECULAR BEAM EPITAXIAL GROWTH MODEL BASED ON THE INVARIANT ENERGY QUADRATIZATION METHOD
}

\author{
XIAOFENG YANG*, JIA ZHAO ${ }^{\dagger}$, AND QI WANG ${ }^{\ddagger}$
}

\begin{abstract}
The Molecular Beam Epitaxial model is derived from the variation of a free energy, that consists of either a fourth order Ginzburg-Landau double well potential or a nonlinear logarithmic potential in terms of the gradient of a height function. One challenge in solving the MBE model numerically is how to develop proper temporal discretization for the nonlinear terms in order to preserve energy stability at the time-discrete level. In this paper, we resolve this issue by developing a first and second order time-stepping scheme based on the "Invariant Energy Quadratization" (IEQ) method. The novelty is that all nonlinear terms are treated semi-explicitly, and the resulted semi-discrete equations form a linear system at each time step. Moreover, the linear operator is symmetric positive definite and thus can be solved efficiently. We then prove that all proposed schemes are unconditionally energy stable. The semi-discrete schemes are further discretized in space using finite difference methods and implemented on GPUs for high-performance computing. Various 2D and 3D numerical examples are presented to demonstrate stability and accuracy of the proposed schemes.
\end{abstract}

Key words. Molecular Beam Epitaxial, Linear, Invariant Energy Quadratization, Unconditional, Energy Stability, Second Order.

AMS subject classifications. $65 \mathrm{~N} 1265 \mathrm{M} 12$ 65M70

1. Introduction. The Molecular Beam Epitaxial (MBE) growth technique is a powerful technique to make compound semiconductor materials with great precision and high purity. It can produce many complex structures of varying layers that are further processed to produce a range of electronic and optoelectronic devices, including high speed transistors, light-emitting diodes, high-efficiency solar cells, and solid state lasers. It has a wide range of applications in Digital Economy, Energy, and Semiconductor Nanotechnology research fields, which motivate people to develop mathematical models as well as simulation tools to understand the primary mechanisms behind the technology.

So far quite a few mathematical models as well as the accompanying numerical simulation tools have been developed to study dynamics of the MBE growth process. These include atomistic models which are implemented in the form of molecular dynamics or kinetic Monte Carlo simulations (cf. [5,14,20]); continuum models which are based on partial differential equations and are appropriate mainly for investigating the temporal evolution of MBE instability at large time and length scales (cf. [16, 26]); and hybrid models that can be considered as a compromise between atomistic models and continuum ones (cf. $[1,12]$ ). In this paper, we consider numerical approximations to the continuum MBE growth model, which is obtained by minimizing the given free energy where the nonlinear potential is either a fourth order Ginzburg-Landau double well potential or a nonlinear logarithmic potential in terms of the gradient of a height function.

A feature of the continuum MBE model considered in this paper is that it is derived from a

*Corresponding author, Department of Mathematics, University of South Carolina, Columbia, SC 29208, USA. Email: xfyang@math.sc.edu. This author's research is partially supported by the U.S. National Science Foundation under grant numbers DMS-1200487 and DMS-1418898, and the U.S. Air Force Office of Scientific Research under grant number FA9550-12-1-0178.

${ }^{\dagger}$ Department of Mathematics, University of South Carolina, Columbia, SC 29208, USA; Department of Mathematics, University of North Carolina at Chapel Hill, Chapel Hill, NC, 27599, USA; Email: zhao62@math.sc.edu. This author's research is partially supported by a ASPIRE grant from The Office of the Vice President for Research University of South Carolina.

¥Department of Mathematics, University of South Carolina, Columbia, SC 29208; Beijing Computational Science Research Center, Beijing, China; School of Materials Science and Engineering, Nankai University, Tianjin, China; Email: qwang@math.sc.edu. This author's research is partially supported by the U.S. National Science Foundation under grant number DMS-1200487, DMS-1517347, the U.S. Air Force Office of Scientific Research under grant number FA9550-12-10178, and the SC EPSCOR GEAR award. 
variational approach and thereby satisfies an energy dissipation law. The energy dissipation law in fact serves as a guidance for the design of thermodynamically consistent (energy stable) numerical schemes since it is especially desirable in the design of numerical schemes that preserve the energy dissipation property at the discrete level. On the one hand, the preservation of the energy law irrespectively of the coarseness of the discretization is critical for the numerical schemes to capture correct long time dynamics of the system. On the other hand, unconditional stability of the energy dissipation preserving schemes provides flexibility for dealing with the stiffness issue in the model.

There have been many existing attempts to develop efficient numerical schemes for solving the MBE model, for which the main challenge lies in how to find proper approaches to discretize the nonlinear terms while preserving the energy stability. We recall that there are two prevalent numerical techniques for doing this. The first one is the stabilization approach, where the term from the nonlinear potential is simply treated explicitly and some linear stabilizing terms are added to improve the stability. Such linear scheme is extremely easy to implement, however, it actually fails to preserve the energy law since the magnitude of the stabilizing term has to depend on the currently undetermined numerical solution (cf. [29]). The second technique is the convex splitting approach (cf. [2, 9, 22, 27, 28]), where the convex part of the potential is treated implicitly while the concave part is treated explicitly. The convex splitting approach is unconditionally energy stable, however, it produces nonlinear schemes in most cases so that the implementation is relatively complicated and the computational cost is relatively high, if compared with the linear approach in [29]. An exceptional case is in [2], where the authors developed a linear scheme to solve the MBE model for the case without slope selection by combining the stabilization approach with the convex splitting approach altogether. Although the scheme is very easy to implement and unconditionally energy stable, it is first order accurate in time.

In this paper, we aim to develop second order, linear, unconditionally energy stable numerical schemes to solve the MBE model. Instead of considering to design special linear stabilizers or analyzing the convexity of nonlinear functionals, we adopt the newly developed Invariant Energy Quadratization (IEQ) method, which is a novel approach but had been successfully applied to solve various phase field models with polynomial type potentials (cf. [11,31,33,34]). For the MBE model, the application of the IEQ method faces new challenges due to the special forms of nonlinear terms, e.g., the double well potential but in terms of the gradient, and the logarithmic type potential. The essential idea of the IEQ method is to transform the free energy into a quadratic (since the nonlinear potential is usually bounded from below) function of a set of new variables via a change of variables. Then, for the reformulated model in terms of the new variables that still retains the identical energy dissipation law, all nonlinear terms can be treated semi-explicitly, which then yields a linear system. As a result, for the MBE model, we develop a set of efficient schemes which are accurate (up to second order in time), easy-to-implement (linear system), and unconditionally energy stable (with a discrete energy dissipation law). We rigorously prove unconditional energy stability for all proposed schemes, including the first order backward Euler, the second order Adam-Bashforth, and the second order Crank-Nicolson schemes. Moreover, we show that the linear operator of the system for all proposed schemes are symmetric positive definite, so that one can solve it using the well-developed fast matrix solvers efficiently (CG or other Krylov subspace methods). Through a set of benchmarking 2D and 3D simulations, we demonstrate stability and accuracy of the proposed schemes thereafter.

The rest of the paper is organized as follows. In Section 2, we present the MBE model and its energy dissipation property. In Section 3, we develop the numerical schemes and prove their unconditional energy stability and unique solvability in the semi-discretized case in time. In Section 4, we present some numerical simulations to demonstrate the accuracy and efficiency of the proposed schemes. Finally, some concluding remarks are presented in Section 5. 
2. MBE Models. For convince of presentation, we fixed some notation in the paper. Consider a confined domain $\Omega i n \mathbb{R}^{d}, d=2,3$. We denote the $L^{2}$ inner product between functions $f(\boldsymbol{x})$ and $g(\boldsymbol{x})$ by

$$
(f(\boldsymbol{x}), g(\boldsymbol{x}))=\int_{\Omega} f(\boldsymbol{x}) g(\boldsymbol{x}) d \boldsymbol{x},
$$

with $\boldsymbol{x} \in \mathbb{R}^{d}$, and the $L^{2}$ norm of function $f(x)$ by

$$
\|f\|=(f, f) .
$$

We present the MBE growth model and discuss its energy dissipation properties, which serve as a guideline for us developing numerical schemes. Let $\phi(\boldsymbol{x}, t)$ be the epitaxy surface height with $\boldsymbol{x} \in \Omega$, where $\Omega$ is a confined domain $\in \mathbb{R}^{d}, d=2,3$. Under typical conditions for MBE growth, the height evolution equation is given in a relaxation dynamical form (see, e.g., [18]):

$$
\phi_{t}=-M \mu,
$$

where $M$ is the mobility coefficient and $\mu=\frac{\delta E}{\delta \phi}$ is the variational derivative of the Ehrlich-Schwoebel energy $E$ (cf. $[8,21])$, given by

$$
E(\phi)=\int_{\Omega}\left(\frac{\epsilon^{2}}{2}(\Delta \phi)^{2}+F(\nabla \phi)\right) d \boldsymbol{x},
$$

where $F(\mathbf{s})$ is a nonlinear, smooth function of its argument $\mathbf{s} \in R^{d}, \epsilon$ is a constant (inversely proportional to the size of the system). The first term in the free energy represents the surface diffusion effect and the second term represents a continuum description of the Ehrlich-Schwoebel effect which means that the adatoms (absorbed atoms) must overcome a higher energy barrier to stick to a step from an upper rather than from a lower terrace.

There are two popular choices of the nonlinear bulk potential $F(\nabla \phi)$ :

(i) Double well potential, for the case of slope selection model (denoted by "the Slope-Model"),

$$
F_{A}(\nabla \phi)=\frac{1}{4}\left(|\nabla \phi|^{2}-1\right)^{2}
$$

(ii) Logrithmic potential, for the case of without slope selection (denoted by "the No-Slope-Model"),

$$
F_{B}(\nabla \phi)=-\frac{1}{2} \ln \left(1+|\nabla \phi|^{2}\right) .
$$

Thus, the governing equation for the Slope-Model is given by

$$
\phi_{t}=-M \frac{\delta E(\phi)}{\delta \phi}=-M\left(\epsilon^{2} \Delta^{2} \phi-\nabla \cdot\left(\left(|\nabla \phi|^{2}-1\right) \nabla \phi\right)\right),
$$

while the governing equation for the No-Slope-Model reads as follows,

$$
\phi_{t}=-M \frac{\delta E(\phi)}{\delta \phi}=-M\left(\epsilon^{2} \Delta^{2} \phi+\nabla \cdot\left(\frac{1}{1+|\nabla \phi|^{2}} \nabla \phi\right)\right) .
$$

With the periodic boundary condition or any other proper boundary condition that can satisfy the flux free condition at the boundary $\left.\partial_{\boldsymbol{n}} \phi\right|_{\partial \Omega}=0$ and $\left.\partial_{\boldsymbol{n}} \Delta \phi\right|_{\partial \Omega}=0$, we have the mass conservation 
property

$$
\frac{d}{d t} \int_{\Omega} \phi(\boldsymbol{x}, t) d \boldsymbol{x}=0
$$

where $\boldsymbol{n}$ is the outward normal on the boundary.

The bulk energy functional $F_{A}(\nabla \phi)$ in (2.5) for the Slope-Model may be viewed as an approximation of the bulk energy $F_{B}(\nabla \phi)$ in (2.6) for the No-Slope-Model, under the assumption that the gradient of the height $(\nabla \phi)$ is small (cf. [17]), e.g.,

$$
\frac{1}{1+|\nabla \phi|^{2}} \approx 1-|\nabla \phi|^{2}
$$

$F_{A}(\nabla \phi)$ can also drive $|\nabla \phi| \rightarrow 1$ where a slope selection mechanism is embedded. Thus, these are significant differences for the long time coarsening processes between these two energy functionals.

One can easily obtain the energy dissipation property for the two models mentioned above. In details, for the Slope-Model, by taking the $L^{2}$ inner product of $(2.7)$ with $-\frac{1}{M} \phi_{t}$, we obtain

$$
\frac{d}{d t} E_{A}(\phi)=-\frac{1}{M}\left\|\phi_{t}\right\|^{2} \leq 0
$$

where

$$
E_{A}(\phi)=\int_{\Omega}\left(\frac{\epsilon^{2}}{2}(\Delta \phi)^{2}+\frac{1}{4}\left(|\nabla \phi|^{2}-1\right)^{2}\right) d \boldsymbol{x}
$$

For the No-Slope-Model, by taking the inner product with the same test function $-\frac{1}{M} \phi_{t}$, we obtain

$$
\frac{d}{d t} E_{B}(\phi)=-\frac{1}{M}\left\|\phi_{t}\right\|^{2} \leq 0
$$

where

$$
E_{B}(\phi)=\int_{\Omega}\left(\frac{\epsilon^{2}}{2}(\Delta \phi)^{2}-\frac{1}{2} \ln \left(1+|\nabla \phi|^{2}\right)\right) d \boldsymbol{x}
$$

In both cases, the energy dissipation rate density is given by $-\frac{1}{M}\left\|\phi_{t}\right\|^{2}$, though $\phi_{t}$ are different.

2.1. Equivalent PDE system and its new energy dissipation law. We introduce two auxiliary functions $U(\phi)$ and $V(\phi)$ in terms of $\phi$ with the following definitions,

$$
\begin{aligned}
U(\phi) & =|\nabla \phi|^{2}-1, \\
V(\phi) & =\sqrt{\ln \left(1+|\nabla \phi|^{2}\right)+A},
\end{aligned}
$$

where $A$ is a positive constant such that $\ln \left(1+x^{2}\right)+A>0$ for any $x \in \mathbb{R}$, for example, $A=1$. Then, the free energy (2.12) for the Slope-Model is transformed into a quadratic function of $\phi$ and $U$ :

$$
E_{A}(\phi, U)=\int_{\Omega}\left(\frac{\epsilon^{2}}{2}(\Delta \phi)^{2}+\frac{1}{4} U^{2}\right) d \boldsymbol{x},
$$


and the free energy (2.14) for the No-Slope-Model is transformed into the following form,

$$
E_{B}(\phi, V)=\int_{\Omega}\left(\frac{\epsilon^{2}}{2}(\Delta \phi)^{2}-\frac{1}{2} V^{2}+\frac{1}{2} A\right) d \boldsymbol{x} .
$$

For the Slope-Model, we have the equivalent PDE system that reads as follows:

$$
\begin{aligned}
\phi_{t} & =-M\left(\epsilon^{2} \Delta^{2} \phi-\nabla \cdot(U \nabla \phi)\right), \\
U_{t} & =2 \nabla \phi \cdot \nabla \phi_{t},
\end{aligned}
$$

where (2.20) is obtained by taking the time derivative for the new variable $U$. The equations (2.19)(2.20) form a closed system with the initial conditions

$$
\left\{\begin{array}{l}
\phi(t=0)=\phi_{0} \\
U(t=0)=\left|\nabla \phi_{0}\right|^{2}-1 .
\end{array}\right.
$$

Similarly, for the No-Slope-Model, the equivalent PDE system reads as follows,

$$
\begin{aligned}
\phi_{t} & =-M\left(\epsilon^{2} \Delta^{2} \phi+\nabla \cdot(V \mathbf{H})\right), \\
V_{t} & =\mathbf{H} \cdot \nabla \phi_{t},
\end{aligned}
$$

where

$$
\mathbf{H}=\frac{\nabla \phi}{\left(1+|\nabla \phi|^{2}\right) \sqrt{\ln \left(1+|\nabla \phi|^{2}\right)+A}}
$$

with the initial conditions

$$
\left\{\begin{array}{l}
\phi(t=0)=\phi_{0} \\
V(t=0)=\sqrt{\ln \left(1+\left|\nabla \phi_{0}\right|^{2}\right)+A}
\end{array}\right.
$$

The boundary conditions for both models are same as the old system, i.e., either periodic boundary conditions or the no-flux boundary condition as $\left.\partial_{\mathbf{n}} \phi\right|_{\partial \Omega}=\left.\partial_{\mathbf{n}} \Delta \phi\right|_{\partial \Omega}=0$. We remark that the equations for $U$ and $V$ do not need boundary conditions since they are just ODEs with respect to the time.

The new system still retains a similar energy law, but it is in terms of the new variables now. For the system (2.19)-(2.20) of the Slope-Model, we take the $L^{2}$ inner product of (2.19) with $-\frac{1}{M} \phi_{t}$, of (2.20) with $\frac{1}{2} U$, and perform integration by parts. By combining all terms together, we obtain the energy dissipation law,

$$
\frac{d}{d t} E_{A}(\phi, U)=-\frac{1}{M}\left\|\phi_{t}\right\|^{2} \leq 0
$$

For the system (2.22)-(2.23) of the No-Slope-Model, we take the $L^{2}$ inner product of $(2.22)$ with $-\frac{1}{M} \phi_{t}$, of $(2.23)$ with $V$, and perform integration by parts. Combining all terms together, we obtain the energy dissipation law,

$$
\frac{d}{d t} E_{B}(\phi, V)=-\frac{1}{M}\left\|\phi_{t}\right\|^{2} \leq 0
$$

Several remarks are in order. 
Remark 2.1. We emphasize that the new transformed PDE system (2.19)-(2.20) and (2.22)-(2.23) are equivalent to the original system (2.7) and (2.8), respectively, since (2.15) and (2.16) can be obtained easily by integrating (2.20) and (2.23) with respect to the time. Therefore, we will develop energy stable numerical schemes for time stepping of the transformed system (2.19)-(2.20) and (2.22)- (2.23). The proposed schemes thereby follow the new energy dissipation law (2.26) and (2.27) instead of the energy law for the originated system (2.11) and (2.13). For the continuous case, the energy law (2.26) and (2.27) for the transformed system are exactly the same as the energy law (2.11) and (2.13) for the original system, respectively. In addition, we remark that, for the discrete case, the energy $E(\phi, U)$ or $E(\phi, V)$ in terms of the new variables can recover the original energy $E(\phi)$ (defined in (2.11) and (2.13)) up to the specified order of the schemes (cf. the detailed argument in Remark 3.2).

Remark 2.2. We add a zero term $-\frac{1}{2} A+\frac{1}{2} A$ in the free energy of $E_{B}(\phi, V)$, where the first $-\frac{1}{2} A$ is combined with the logarithmic function to make the new variable $V$ nonzero. This trick helps to avoid the occurrence of singularities in $\mathbf{H}($ if $|\nabla \phi|=0)$. Such reformulation by adding a zero term does not introduce any additional splitting errors since the added term is in terms of constants. We note that a similar technique has been used in [2] to produce a linear scheme, where a zero term $\Delta \phi-\Delta \phi$ is added, and the minuend is treated implicitly and the subtractor is treated explicitly, that is quite similar to the stabilization approach [24, 29,30]. However, such treatment induces extra splitting errors to spoil the accuracy to an extent, indeed.

3. Numerical Schemes. We now develop semi-discrete numerical schemes for the Slope-Model (2.7) and the No-Slope-Model (2.8), respectively, and show that they retain energy stability properties at the semi-discrete level.

3.1. First-order Scheme. We present the first order scheme to solve the Slope-Model (2.19)(2.20) and the No-Slope-Model (2.22)-(2.23), respectively.

3.1.1. First-order Scheme for the Slope-Model. Assuming that $\phi^{n}, U^{n}$ are already calculated, we then compute $\phi^{n+1}$ and $U^{n+1}$ to solve the Slope-Model (2.19)-(2.20) from the following temporal discrete system:

$$
\begin{aligned}
& \frac{\phi^{n+1}-\phi^{n}}{\delta t}=-M\left(\epsilon^{2} \Delta^{2} \phi^{n+1}-\nabla \cdot\left(U^{n+1} \nabla \phi^{n}\right)\right), \\
& \frac{U^{n+1}-U^{n}}{\delta t}=2 \nabla \phi^{n} \cdot \nabla \frac{\phi^{n+1}-\phi^{n}}{\delta t},
\end{aligned}
$$

with the initial conditions

$$
\left\{\begin{array}{l}
\phi^{0}=\phi_{0}, \\
U^{0}=\left|\nabla \phi_{0}\right|^{2}-1 .
\end{array}\right.
$$

The boundary conditions are either periodic or the no-flux boundary conditions

$$
\left.\partial_{\mathbf{n}} \phi^{n+1}\right|_{\partial \Omega}=\left.\partial_{\mathbf{n}} \Delta \phi^{n+1}\right|_{\partial \Omega}=0 .
$$

Since (3.2) is actually an equality, thus we can rewrite it as

$$
U^{n+1}=\alpha_{1}^{n}+2 \nabla \phi^{n} \cdot \nabla \phi^{n+1},
$$

where

$$
\alpha_{1}^{n}=U^{n}-2\left|\nabla \phi^{n}\right|^{2} .
$$


Then the schemes (3.1)-(3.2) can be rewritten as

$$
\mathbb{A}_{1}\left(\phi^{n+1}\right)=\mathbb{B}_{1}^{n}
$$

where

$$
\mathbb{A}_{1}(\phi)=\frac{1}{M \delta t} \phi-2 \nabla \cdot\left(\left(\nabla \phi^{n} \cdot \nabla \phi\right) \nabla \phi^{n}\right)+\epsilon^{2} \Delta^{2} \phi, \quad \mathbb{B}_{1}^{n}=\nabla \cdot\left(\alpha_{1}^{n} \nabla \phi^{n}\right)+\frac{\phi^{n}}{M \delta t} .
$$

Therefore, we can solve $\phi^{n+1}$ directly from (3.7). Namely, the new variable $U$ does not involve any extra computational costs.

We show the well-posedness of the linear system (3.1)-(3.2) (or (3.7)) as follows.

TheOrem 3.1. The linear system (3.1)-(3.2) (or (3.7)) admits a unique solution in $H^{2}(\Omega)$.

Proof. (i). For any $\psi$ satisfy the boundary conditions of the system (periodic or no-flux), using integration by parts, we derive

$$
\begin{aligned}
\left(\mathbb{A}_{1}(\phi), \psi\right) & =\frac{1}{M \delta t}(\phi, \psi)+2\left(\nabla \phi^{n} \cdot \nabla \phi, \nabla \phi^{n} \cdot \nabla \psi\right)+\epsilon^{2}(\Delta \phi, \Delta \psi) \\
& \leq \frac{1}{M \delta t}\|\phi\|\|\psi\|+C_{1}\|\nabla \phi\|\|\nabla \psi\|+\epsilon^{2}\|\Delta \phi\|\|\Delta \psi\| \\
& \leq C_{2}\|\phi\|_{H^{2}}\|\psi\|_{H^{2}} .
\end{aligned}
$$

where $C_{1}=\left\|\nabla \phi^{n}\right\|_{L^{\infty}}$. Therefore, the bilinear form $\left(A_{1}(\phi), \psi\right), \forall \phi, \psi \in H^{2}(\Omega)$ is bounded.

(ii). It is easy to derive that

$$
\left(\mathbb{A}_{1}(\phi), \phi\right)=\frac{1}{M \delta t}\|\phi\|^{2}+2\left\|\nabla \phi^{n} \cdot \nabla \phi\right\|^{2}+\epsilon^{2}\|\Delta \phi\|^{2} \geq \frac{1}{M \delta t}\|\phi\|^{2}+\epsilon^{2}\|\Delta \phi\|^{2} \geq C_{3}\|\phi\|_{H^{2}}^{2} .
$$

The last inequality is due to Gagliardo-Nirenberg inequalities. Thus the bilinear form $\left(A_{1}(\phi), \psi\right)$ is coercive. Then from the Lax-Milgram theorem, we conclude the linear system admits a unique solution in $H^{2}(\Omega)$.

Moreover, we have the following property for the linear operator $\mathbb{A}_{1}$.

THEOREM 3.2. The linear operator of the system (3.1)-(3.2) (or (3.7)) is symmetric positive definite for the variable $\phi^{n+1}$.

Proof. (i). For any $\psi$ satisfy the boundary conditions of the system (periodic or no-flux), using integration by parts, we derive

$$
\left(\psi, \mathbb{A}_{1}(\phi)\right)=\left(\mathbb{A}_{1}(\psi), \phi\right)
$$

thus $\mathbb{A}_{1}$ is symmetric.

(ii). It is easy to derive that

$$
\left(\mathbb{A}_{1}(\phi), \phi\right)=\frac{1}{M \delta t}\|\phi\|^{2}+2\left\|\nabla \phi^{n} \cdot \nabla \phi\right\|^{2}+\epsilon^{2}\|\Delta \phi\|^{2}>0
$$

for any $\phi \neq 0$ and $\phi$ satisfies the boundary conditions. Thus, the operator $\mathbb{A}_{1}$ is symmetric positive definite.

The energy-stable property of scheme (3.1) -(3.2) is given in the following theorem. 
THEOREM 3.3. Scheme (3.1)-(3.2) is unconditionally energy stable and satisfies the following discrete energy dissipation law,

$$
\frac{1}{\delta t}\left(E_{A}^{n+1}-E_{A}^{n}\right) \leq-\frac{1}{M}\left\|\frac{\phi^{n+1}-\phi^{n}}{\delta t}\right\|^{2}
$$

where, for any $n$,

$$
E_{A}^{n}=\int_{\Omega}\left(\frac{\epsilon^{2}}{2}\left(\Delta \phi^{n}\right)^{2}+\frac{1}{4}\left(U^{n}\right)^{2}\right) d \boldsymbol{x}
$$

Proof. Taking the $L^{2}$ inner product of (3.1) with $-\frac{1}{M}\left(\phi^{n+1}-\phi^{n}\right)$, perfroming integration by parts, and applying the following identity

$$
2(a-b, a)=|a|^{2}-|b|^{2}+|a-b|^{2},
$$

we obtain

$$
\begin{aligned}
-\frac{1}{M \delta t}\left\|\phi^{n+1}-\phi^{n}\right\|^{2} & =\frac{\epsilon^{2}}{2}\left(\left\|\Delta \phi^{n+1}\right\|^{2}-\left\|\Delta \phi^{n}\right\|^{2}+\left\|\Delta \phi^{n+1}-\phi^{n}\right\|^{2}\right) \\
& +\left(U^{n+1} \nabla \phi^{n}, \nabla\left(\phi^{n+1}-\phi^{n}\right)\right) .
\end{aligned}
$$

Taking the $L^{2}$ inner product of (3.1) with $-\frac{1}{2} \delta t U^{n+1}$, we obtain

$$
-\frac{1}{4}\left(\left\|U^{n+1}\right\|^{2}-\left\|U^{n}\right\|^{2}+\left\|U^{n+1}-U^{n}\right\|^{2}\right)=-\left(\nabla \phi^{n} \cdot \nabla\left(\phi^{n+1}-\phi^{n}\right), U^{n+1}\right) .
$$

Combining (3.16) and (3.17), we obtain

$$
\begin{aligned}
-\frac{1}{M \delta t}\left\|\phi^{n+1}-\phi^{n}\right\|^{2} & =\frac{1}{4}\left(\left\|U^{n+1}\right\|^{2}-\left\|U^{n}\right\|^{2}+\left\|U^{n+1}-U^{n}\right\|^{2}\right) \\
& +\frac{\epsilon^{2}}{2}\left(\left\|\Delta \phi^{n+1}\right\|^{2}-\left\|\Delta \phi^{n}\right\|^{2}+\left\|\Delta \phi^{n+1}-\Delta \phi^{n}\right\|^{2}\right) .
\end{aligned}
$$

Finally we obtain the desired result (3.13) after dropping some positive terms.

Remark 3.1. One can further introduce an extra auxiliary variable $\psi=\Delta \phi$ to split the fourth order PDE system (3.1) into two second order elliptic equations, all the derivations about the well-posedness and the energy stabilities are essentially the same.

Remark 3.2. The proposed schemes follow the new energy dissipation law (2.26) formally instead of the energy law for the originated system (2.12). In the time-discrete case, the energy $E_{A}\left(\phi^{n+1}, U^{n+1}\right)$ (defined in (3.14)) can be rewritten as a first order approximation to the Lyapunov functionals in $E\left(\phi^{n+1}\right)$ (defined in (2.12)), that can be observed from the following facts, heuristically. From (3.2) we have

$$
U^{n+1}-\left(\left|\nabla \phi^{n+1}\right|^{2}-1\right)=U^{n}-\left(\left|\nabla \phi^{n}\right|^{2}-1\right)+R_{n+1},
$$

where $R_{n+1}=-\left|\nabla\left(\phi^{n+1}-\phi^{n}\right)\right|^{2}$. Since $R_{k}=O\left(\delta t^{2}\right)$ for $0 \leq k \leq n+1$ and $U^{0}=\left|\nabla \phi^{0}\right|^{2}-1$, by mathematical induction we can easily get

$$
U^{n+1}=\left|\nabla \phi^{n+1}\right|^{2}-1+O(\delta t) .
$$


Remark 3.3. Other numerical schemes have been proposed for the the Slope-Model under consideration. In [17], Li and Liu proposed the following numerical scheme

$$
\frac{\phi^{n+1}-\phi^{n}}{\delta t}=\frac{3}{2} \nabla \cdot\left(\nabla \phi^{n} \cdot \nabla \phi^{n} \nabla \phi^{n}\right)-\frac{1}{2} \nabla \cdot\left(\nabla \phi^{n-1} \cdot \nabla \phi^{n-1} \nabla \phi^{n-1}\right)-\frac{\epsilon^{2}}{2}\left(\Delta^{2} \phi^{n+1}+\Delta^{2} \phi^{n}\right) .
$$

The scheme is not expected to be energy stable although it is second order and unconditionally uniquely solvable.

In [29], Xu and Tang proposed the first order stabilization scheme as follows,

$$
\frac{\phi^{n+1}-\phi^{n}}{\delta t}=s\left(\Delta \phi^{n+1}-\Delta \phi^{n}\right)+\nabla \cdot\left(\nabla \phi^{n} \cdot \nabla \phi^{n} \nabla \phi^{n}\right)-\epsilon^{2} \Delta^{2} \phi^{n+1} .
$$

$X u$ and Tang proved that the scheme is energy stable, provided the preassigned constant $s$ is sufficiently large. However, they showed that the appropriate parameter $s$ depends on the unknown $\phi^{n+1}$ and thus the scheme is not unconditionally stable.

In [19], Qiao, Sun and Zhang proposed a linear scheme as follows,

$$
\frac{\phi^{n+1}-\phi^{n}}{\delta t}=\nabla \cdot\left(\nabla \phi^{n} \cdot \nabla \phi^{n} \nabla \phi^{n+1}\right)-\Delta \phi^{n}-\epsilon^{2} \Delta^{2} \phi^{n+1} .
$$

Such linear scheme is uniquely solvable and its computational cost is the same as the our proposed scheme (3.7). However, it is not energy stable although the numerical solution still follows some inequality

$$
\left\|\phi^{n+1}\right\|^{2}-\left\|\phi^{n}\right\|^{2} \leq \pi^{2} \delta t,\left\|\phi^{n+1}\right\|^{2} \leq\left\|\phi^{0}\right\|^{2}+2 \pi^{2} t^{n+1} .
$$

In [27], Wang, Wang and Wise proposed the first order convex splitting scheme as follows,

$$
\frac{\phi^{n+1}-\phi^{n}}{\delta t}=\nabla \cdot\left(\nabla \phi^{n+1} \cdot \nabla \phi^{n+1} \nabla \phi^{n+1}\right)-\Delta \phi^{n}-\epsilon^{2} \Delta^{2} \phi^{n+1} .
$$

The scheme is unconditionally energy stable. Actually, we notice that if the Newton iterative method is applied for solving the nonlinear system, at each iteration (3.25) would yield the same linear system as given by our first order scheme (3.7). Hence the cost of solving our scheme is the same as the costs of performing one iteration of Newton method for the nonlinear scheme, provided that the same linear solvers are applied (for instance multigrid with Gauss-Seidel relaxation). It is clear that our scheme would be much more efficient than the nonlinear convex-splitting schemes.

3.1.2. First-order Scheme for the No-Slope-Model. Assume that $\phi^{n}, V^{n}$ are already calculated, we then compute $\phi^{n+1}$ and $V^{n+1}$ to solve the the No-Slope-Model (2.22)-(2.23) from the following temporal discrete system:

Step1:

$$
\frac{\phi^{n+1}-\phi^{n}}{\delta t}=-M\left(\epsilon^{2} \Delta^{2} \phi^{n+1}+\nabla \cdot\left(V^{n} \mathbf{H}^{n}\right)\right)
$$

with

$$
\mathbf{H}^{n}=\frac{\nabla \phi^{n}}{\left(1+\left|\nabla \phi^{n}\right|^{2}\right) \sqrt{\ln \left(1+\left|\nabla \phi^{n}\right|^{2}\right)+A}} .
$$




\section{Step2:}

$$
\frac{V^{n+1}-V^{n}}{\delta t}=\mathbf{H}^{n} \cdot \nabla \frac{\phi^{n+1}-\phi^{n}}{\delta t}
$$

The initial conditions are

$$
\left\{\begin{array}{l}
\phi^{0}=\phi_{0} \\
V^{0}=\sqrt{\ln \left(1+\left|\nabla \phi_{0}\right|^{2}\right)+A} .
\end{array}\right.
$$

The boundary conditions are periodic or no-flux boundary conditions

$$
\left.\partial_{\mathbf{n}} \phi^{n+1}\right|_{\partial \Omega}=\left.\partial_{\mathbf{n}} \Delta \phi^{n+1}\right|_{\partial \Omega}=0 .
$$

We emphasize that the Step $\mathbf{1}$ and the Step $\mathbf{2}$ are totally decoupled since the nonlinear coefficient $\mathbf{H}$ and the new variable $V$ are both treated explicitly in Step 1. Therefore, the scheme is very easy to implement. The existence and the uniqueness of the solution are obvious. Furthermore, as we will show below, the scheme is unconditionally energy stable.

THEOREM 3.4. The scheme (3.26)-(3.28) is unconditionally stable, and satisfies the following discrete energy dissipation property,

$$
\frac{1}{\delta t}\left(E_{B}^{n+1}-E_{B}^{n}\right) \leq-\frac{1}{M}\left\|\frac{\phi^{n+1}-\phi^{n}}{\delta t}\right\|^{2}
$$

where, for any $n$

$$
E_{B}^{n}=\int_{\Omega}\left(\frac{\epsilon^{2}}{2}\left(\Delta \phi^{n}\right)^{2}-\frac{1}{2}\left(V^{n}\right)^{2}+\frac{1}{2} A\right) d \boldsymbol{x}
$$

Proof. Taking the $L^{2}$ inner product of (3.26) with $-\frac{1}{M}\left(\phi^{n+1}-\phi^{n}\right)$ and performing integration by parts, we obtain

$$
\begin{aligned}
-\frac{1}{M \delta t}\left\|\phi^{n+1}-\phi^{n}\right\|^{2} & =\frac{\epsilon^{2}}{2}\left(\left\|\Delta \phi^{n+1}\right\|^{2}-\left\|\Delta \phi^{n}\right\|^{2}+\left\|\Delta \phi^{n+1}-\Delta \phi^{n}\right\|^{2}\right) \\
& -\left(V^{n} \mathbf{H}^{n}, \nabla\left(\phi^{n+1}-\phi^{n}\right)\right) .
\end{aligned}
$$

Taking the $L^{2}$ inner product of (3.28) with $\delta t V^{n}$, and applying the following identity,

$$
2(a-b, b)=|a|^{2}-|b|^{2}-|a-b|^{2},
$$

we obtain

$$
\frac{1}{2}\left(\left\|V^{n+1}\right\|^{2}-\left\|V^{n}\right\|^{2}-\left\|V^{n+1}-V^{n}\right\|^{2}\right)=\left(\mathbf{H}^{n} \cdot \nabla\left(\phi^{n+1}-\phi^{n}\right), V^{n}\right) .
$$

Combining (3.33) and (3.35), we obtain

$$
\begin{aligned}
-\frac{1}{M \delta t}\left\|\phi^{n+1}-\phi^{n}\right\|^{2} & =\frac{\epsilon^{2}}{2}\left(\left\|\Delta \phi^{n+1}\right\|^{2}-\left\|\Delta \phi^{n}\right\|^{2}+\left\|\Delta \phi^{n+1}-\Delta \phi^{n}\right\|^{2}\right) \\
& \left.-\frac{1}{2}\left(\left\|V^{n+1}\right\|^{2}-\left\|V^{n}\right\|^{2}\right)+\frac{1}{2}\left\|V^{n+1}-V^{n}\right\|^{2}\right) .
\end{aligned}
$$


Finally we obtain the desired result (3.31) after dropping some positive terms.

Remark 3.4. Similar to Remark 3.2, we can write (3.28) as follows,

$$
V^{n+1}-\left(\ln \left(1+\left|\nabla \phi^{n+1}\right|^{2}\right)+A\right)=V^{n}-\left(\ln \left(1+\left|\nabla \phi^{n}\right|^{2}\right)+A\right)+R_{n+1},
$$

where

$$
R_{n+1}=-\left(\ln \left(1+\left|\nabla \phi^{n+1}\right|^{2}\right)+A\right)+\left(\ln \left(1+\left|\nabla \phi^{n}\right|^{2}\right)+A\right)+\mathbf{H}^{n} \cdot \nabla\left(\phi^{n+1}-\phi^{n}\right) .
$$

Note that $R_{n+1}$ is the residue of the Taylor expansion of the function $\ln \left(1+\left|\nabla \phi^{n+1}\right|^{2}\right)+A$ at $t=t^{n}$, thus $R_{k}=O\left(\delta t^{2}\right)$ for $0 \leq k \leq n+1$. By mathematical induction we can easily get

$$
V^{n+1}=\left(\ln \left(1+\left|\nabla \phi^{n+1}\right|^{2}\right)+A\right)+O(\delta t) .
$$

Remark 3.5. There are a few unconditionally energy stable schemes that are developed based on the convex splitting approach. In [27], Wang, Wang and Wise proposed a nonlinear first order convex splitting scheme as follows,

$$
\frac{\phi^{n+1}-\phi^{n}}{\delta t}=-\epsilon^{2} \Delta^{2} \phi^{n+1}-\nabla \cdot\left(\frac{\nabla \phi^{n+1}}{1+\left|\nabla \phi^{n+1}\right|^{2}}\right)+\Delta \phi^{n+1}-\Delta \phi^{n} .
$$

where the nonlinear logarithmic term is treated implicitly. The scheme is unconditionally energy stable and uniquely solvable. It is developed based on the following convex-concave combinations of the free energy,

$$
E=\int_{\Omega}\left(\frac{1}{2}|\nabla \phi|^{2}-\ln \left(1+|\nabla \phi|^{2}\right)+\frac{\epsilon^{2}}{2}|\Delta \phi|^{2}\right) d \boldsymbol{x}-\int_{\Omega} \frac{1}{2}|\nabla \phi|^{2} d \boldsymbol{x}
$$

In contrast, in [2], Chen, Conde, Wang, Wang, and Wise proposed a more efficient, linear first order scheme as follows, which is also based on the convex splitting approach,

$$
\frac{\phi^{n+1}-\phi^{n}}{\delta t}=-\epsilon^{2} \Delta^{2} \phi^{n+1}-\nabla \cdot\left(\frac{\nabla \phi^{n}}{1+\left|\nabla \phi^{n}\right|^{2}}\right)+\Delta \phi^{n+1}-\Delta \phi^{n} .
$$

The scheme is unconditionally energy stable, and the development is based on the following convexconcave combinations of the free energy,

$$
E=\int_{\Omega}\left(\frac{1}{2} \epsilon^{2}(\Delta \phi)^{2}+\frac{1}{2}|\nabla \phi|^{2}\right) d \boldsymbol{x}-\int_{\Omega}\left(\frac{1}{2}|\nabla \phi|^{2}+\ln \left(1+|\nabla \phi|^{2}\right)\right) d \boldsymbol{x}
$$

We remark that the computational cost of solving such linear scheme is the same as the cost of our scheme (3.26)-(3.28).

Remark 3.6. We notice that the idea of the IEQ approach is very simple but quite different from the traditional time marching schemes. For example, it does not require the convexity as the convex splitting approach (cf. [9]) or the boundness for the second order derivative as the stabilization approach (cf. [23, 29]). Through a simple substitution of new variables, the complicated nonlinear potentials are transformed into quadratic forms. We summarize the great advantages of this quadratic transformations as follows: (i) this quadratization method works well for various complex nonlinear terms as long as the corresponding nonlinear potentials are bounded from below; (ii) the complicated nonlinear potential is transferred to a quadratic polynomial form which is much easier to handle; (iii) the derivative of the 
quadratic polynomial is linear, which provides the fundamental support for linearization method; (iv) the quadratic formulation in terms of new variables can automatically maintain this property of positivity (or bounded from below) of the nonlinear potentials.

Remark 3.7. We thank the anonymous reviewer for pointing out, the exactly same scheme (3.1)(3.2) and (3.57)-(3.58) had been developed in Chen's thesis [4] (in Chinese) for the Slope-Model. In [4], to solve the Slope-Model, Chen actually adopted the so-called Lagrange multiplier method that was developed in [11,25] for solving the Cahn-Hilliard equation with double well potential in which, the nonlinear potential is $F(\phi)=\left(\phi^{2}-1\right)^{2}$. The application of the Lagrange multiplier method to solve the Slope-Model is very natural since the nonlinear potential for the Slope-Model is still the double well type potential polynomial, i.e., $F(\phi)=\left(|\nabla \phi|^{2}-1\right)^{2}$.

We remark that when the nonlinear potential takes the fourth order polynomial type, e.g. $F(\psi)=$ $\left(\psi^{2}-1\right)^{2}$ where $\psi=\phi$ for Cahn-Hilliard model and $\psi=|\nabla \phi|$ for the Slope-Model, this IEQ method is exactly the same as the Lagrange multiplier method in [4,11,25]. But the Lagrange multiplier method will only work the fourth order polynomial type potential since its derivative $\psi^{3}$ can be decomposed into $\lambda(\psi) \psi$ with $\lambda(\psi)=|\psi|^{2}$ which can be viewed as a Lagrange multiplier term. However, for other type potentials, the Lagrange multiplier method is not applicable. About the application of the IEQ approach to handle other type of nonlinear potentials, e.g., the logarithmic Flory-Huggins potential, or anisotropic gradient entropy, etc., we refer to the authors' other work in [31,33, 34].

In order to present a full set of linear schemes for solving the Slope-Model and the No-Slope-Model, we still keep (3.1)-(3.2) and (3.57)-(3.58) in this paper for the reason of consistency. In addition, we establish the property of symmetric positive definite for the linear operator, and provide the second order BDF2 version scheme for the Slope-Model.

Remark 3.8. For the numerical schemes proposed in this paper, the energy stability is formally derived. The error analysis is rather natural and standard. For the slope model case, notice that $\left\|\Delta \phi^{n+1}\right\|$ is bounded in Theorem 3.3, one can easily obtain $H^{2}$ bound for the numerical solution from the Poincare inequality since the mass is conserved. Hence, the corresponding convergence analysis can be further carried out. We will implement the rigorous error analysis in the future work that will follow the same lines as the literatures [6, 7, 10,23].

For the the No-Slope-Model, the energy stability result with respect to the modified energy (3.31) (or (3.65) in the second order scheme) does not indicate the $H^{2}$ stability of the numerical solution due to the introduction of the new variable. In comparison with convex splitting approach or other energy stable approach, this stability result is somehow weaker at the theoretical level. Some future work will be further implemented to address this fact.

3.2. Second order schemes. Next, we construct the second order time stepping schemes based on the second order Adam-Bashforth backward differentiation formulas (BDF2) and the Crank-Nicolson $(\mathrm{CN})$ formulas.

3.2.1. Second-order Scheme for the Slope-Model. Assume that we have found $\phi^{n}, U^{n}$ and $\phi^{n-1}, U^{n-1}$, then we compute $\phi^{n+1}, U^{n+1}$ to solve the Slope-Model (2.19)-(2.20) as follows,

$$
\begin{aligned}
& \frac{3 \phi^{n+1}-4 \phi^{n}+\phi^{n-1}}{2 \delta t}=-M\left(\epsilon^{2} \Delta^{2} \phi^{n+1}-\nabla \cdot\left(U^{n+1} \nabla \phi^{\star}\right)\right), \\
& \frac{3 U^{n+1}-4 U^{n}+U^{n-1}}{2 \delta t}=2 \nabla \phi^{\star} \cdot \nabla \frac{3 \phi^{n+1}-4 \phi^{n}+\phi^{n-1}}{2 \delta t},
\end{aligned}
$$

where $\phi^{\star}=2 \phi^{n}-\phi^{n-1}$. 
The boundary conditions are either periodic or no-flux boundary conditions

$$
\left.\partial_{\mathbf{n}} \phi^{n+1}\right|_{\partial \Omega}=\left.\partial_{\mathbf{n}} \Delta \phi^{n+1}\right|_{\partial \Omega}=0 .
$$

Similar to the first order schemes, we can rewrite (3.45) as

$$
U^{n+1}=\alpha_{2}^{n}+2 \nabla \phi^{\star} \cdot \nabla \phi^{n+1},
$$

where

$$
\alpha_{2}^{n}=\frac{4 U^{n}-U^{n-1}}{3}-2 \nabla \phi^{\star} \cdot \nabla \frac{4 \phi^{n}-\phi^{n-1}}{3} .
$$

In turn, (3.44) can be rewritten as

$$
\mathbb{A}_{2}\left(\phi^{n+1}\right)=\mathbb{B}_{2}
$$

where

$$
\mathbb{A}_{2}(\phi)=\frac{3}{2 M \delta t} \phi-2 \nabla \cdot\left(\left(\nabla \phi^{\star} \cdot \nabla \phi\right) \nabla \phi^{\star}\right)+\epsilon^{2} \Delta^{2} \phi, \quad \mathbb{B}_{2}=\nabla \cdot\left(\alpha_{2}^{n} \nabla \phi^{\star}\right)+\frac{4 \phi^{n}-\phi^{n-1}}{2 M \delta t} .
$$

Therefore, we can solve $\phi^{n+1}$ directly from (3.49). Once we obtain $\phi^{n+1}, U^{n+1}$ is automatically given in (3.47). Namely, the new variables $U$ do not involve any extra computational cost.

We show the well-posedness of the linear system (3.49) as follows.

TheOREM 3.5. The linear system (3.49) (or (3.44)-(3.45)) admits a unique solution. Moreover, the linear opeartor is symmetric positive definite.

Proof. The proof is similar to Theorem 3.1 and 3.2, thus we omit the details here.

TheOREm 3.6. Scheme (3.44)-(3.45) is unconditionally stable and satisfies the following discrete energy dissipation law,

$$
\frac{1}{\delta t}\left(E_{A}^{n+1, n}-E_{A}^{n, n-1}\right) \leq-\frac{1}{M}\left\|\frac{3 \phi^{n+1}-4 \phi^{n}+\phi^{n-1}}{2 \delta t}\right\|^{2},
$$

where

$$
E_{A}^{n+1, n}=\int_{\Omega}\left\{\frac{\epsilon^{2}}{2}\left(\frac{\left(\Delta \phi^{n+1}\right)^{2}+\left(2 \Delta \phi^{n+1}-\Delta \phi^{n}\right)^{2}}{2}\right)+\frac{1}{4}\left(\frac{\left(U^{n+1}\right)^{2}+\left(2 U^{n+1}-U^{n}\right)^{2}}{2}\right)\right\} d \boldsymbol{x}
$$

Proof. Taking the $L^{2}$ inner product of (3.44) with $-\frac{1}{2 M}\left(3 \phi^{n+1}-4 \phi^{n}+\phi^{n-1}\right)$, and applying the following identity,

$$
2(3 a-4 b+c, a)=\left(|a|^{2}+|2 a-b|^{2}\right)-\left(|b|^{2}+|2 b-c|^{2}\right)+|a-2 b+c|^{2},
$$


we obtain

$$
\begin{aligned}
-\frac{1}{4 M \delta t}\left\|3 \phi^{n+1}-4 \phi^{n}+\phi^{n-1}\right\|^{2} & =\frac{\epsilon^{2}}{4}\left(\left\|\Delta \phi^{n+1}\right\|^{2}+\left\|2 \Delta \phi^{n+1}-\Delta \phi^{n}\right\|^{2}\right) \\
& -\frac{\epsilon^{2}}{4}\left(\left\|\Delta \phi^{n}\right\|^{2}+\left\|2 \Delta \phi^{n}-\Delta \phi^{n-1}\right\|^{2}\right) \\
& +\frac{\epsilon^{2}}{4}\left\|\Delta \phi^{n+1}-2 \Delta \phi^{n}+\Delta \phi^{n-1}\right\|^{2} \\
& +\frac{1}{2}\left(U^{n+1} \nabla \phi^{\star}, \nabla\left(3 \phi^{n+1}-4 \phi^{n}+\phi^{n-1}\right)\right) .
\end{aligned}
$$

Taking the $L^{2}$ inner product of (3.45) with $-\frac{\delta t}{2} U^{n+1}$, we obtain

$$
\begin{aligned}
& -\frac{1}{8}\left(\left\|U^{n+1}\right\|^{2}+\left\|2 U^{n+1}-U^{n}\right\|^{2}\right)+\frac{1}{8}\left(\left\|U^{n}\right\|^{2}+\left\|2 U^{n}-U^{n-1}\right\|^{2}\right) \\
& -\frac{1}{8}\left\|U^{n+1}-2 U^{n}+U^{n-1}\right\|^{2}=-\frac{1}{2}\left(\nabla \phi^{\star} \cdot \nabla\left(3 \phi^{n+1}-4 \phi^{n}+\phi^{n-1}\right), U^{n+1}\right) .
\end{aligned}
$$

Combining (3.54) and (3.55), we obtain

$$
\begin{aligned}
\frac{1}{8}\left(\left\|U^{n+1}\right\|^{2}+\right. & \left.\left\|2 U^{n+1}-U^{n}\right\|^{2}\right)-\frac{1}{8}\left(\left\|U^{n}\right\|^{2}+\left\|2 U^{n}-U^{n-1}\right\|^{2}\right) \\
& +\frac{\epsilon^{2}}{4}\left(\left\|\Delta \phi^{n+1}\right\|^{2}+\left\|2 \Delta \phi^{n+1}-\Delta \phi^{n}\right\|^{2}\right)-\frac{\epsilon^{2}}{4}\left(\left\|\Delta \phi^{n}\right\|^{2}+\left\|2 \Delta \phi^{n+1}-\Delta \phi^{n}\right\|^{2}\right) \\
& +\frac{1}{8}\left\|U^{n+1}-2 U^{n}+U^{n-1}\right\|^{2}+\frac{\epsilon^{2}}{4}\left\|\Delta \phi^{n+1}-2 \Delta \phi^{n}+\Delta \phi^{n-1}\right\|^{2} \\
& =-\frac{1}{4 M \delta t}\left\|3 \phi^{n+1}-4 \phi^{n}+\phi^{n-1}\right\|^{2} .
\end{aligned}
$$

Finally we obtain the desired result (3.51) after dropping some positive terms.

Remark 3.9. Heuristically, the $\frac{1}{\delta t}\left(E_{A}^{n+1, n}-E_{A}^{n, n-1}\right)$ is a second order approximation of $\frac{d}{d t} E_{A}(\phi, U)$ at $t=t^{n+1}$. For instance, for any smooth variable $S$ with time, one can write

$$
\begin{aligned}
\left(\frac{\left\|S^{n+1}\right\|^{2}+\left\|2 S^{n+1}-S^{n}\right\|^{2}}{2 \delta t}\right) & -\left(\frac{\left\|S^{n}\right\|^{2}+\left\|2 S^{n}-S^{n-1}\right\|^{2}}{2 \delta t}\right) \\
& \cong\left(\frac{\left\|S^{n+2}\right\|^{2}-\left\|S^{n}\right\|^{2}}{2 \delta t}\right)+O\left(\delta t^{2}\right) \cong \frac{d}{d t}\left\|S\left(t^{n+1}\right)\right\|^{2}+O\left(\delta t^{2}\right) .
\end{aligned}
$$

One can easily develop the second order version of the Crank-Nicolson scheme as follows,

$$
\begin{aligned}
& \frac{\phi^{n+1}-\phi^{n}}{\delta t}=-M\left(\epsilon^{2} \Delta^{2} \frac{\phi^{n+1}+\phi^{n}}{2}-\nabla \cdot\left(\frac{U^{n+1}+U^{n}}{2} \nabla \phi^{\dagger}\right)\right), \\
& \frac{U^{n+1}-U^{n}}{\delta t}=-2 \nabla \phi^{\dagger} \cdot \nabla \frac{\phi^{n+1}-\phi^{n}}{\delta t},
\end{aligned}
$$

where $\phi^{\dagger}=\frac{3}{2} \phi^{n}-\frac{1}{2} \phi^{n-1}$.

Its unconditional energy stability is presented as follows with the similar proof as the BDF2 scheme.

THEOREM 3.7. The scheme (3.57)-(3.58) is unconditionally stable, and satisfies the following dis- 
crete energy dissipation law,

$$
\frac{1}{\delta t}\left(E_{A}^{n+1}-E_{A}^{n}\right)=-\frac{1}{M}\left\|\frac{\phi^{n+1}-\phi^{n}}{\delta t}\right\|^{2},
$$

where

$$
E_{A}^{n}=\int_{\Omega}\left(\frac{\epsilon^{2}}{2}\left(\Delta \phi^{n}\right)^{2}+\frac{1}{4}\left(U^{n}\right)^{2}\right) d \boldsymbol{x} .
$$

The proof is similar to the BDF2 scheme and therefore omitted. We emphasize the discrete energy of the Crank-Nicolson scheme strictly follows the PDE energy law (2.26) (i.e., "=" instead of " $\leq$ ") .

Remark 3.10. In [22], Shen et. al proposed the second order convex splitting scheme as follows,

$$
\begin{aligned}
\frac{\phi^{n+1}-\phi^{n}}{\delta t} & \left.=\frac{1}{4} \nabla \cdot\left(\left|\nabla \phi^{n+1}\right|^{2}+\left|\nabla \phi^{n}\right|^{2}\right)\left(\nabla \phi^{n+1}+\nabla \phi^{n}\right)\right) \\
& -\Delta\left(\frac{3}{2} \phi^{n}-\frac{1}{2} \phi^{n-1}\right)-\frac{\epsilon^{2}}{2} \Delta^{2}\left(\phi^{n+1}+\phi^{n}\right)
\end{aligned}
$$

The scheme is unconditionally energy stable but nonlinear. When using the Newton iterative method for solving the nonlinear system, at each iteration, the yielded linear system is similar to our linear scheme of (3.49). Therefore, as the first order convex splitting scheme (3.25), the cost of solving our scheme is (3.49) the same as the cost of performing one iteration of Newton method for the nonlinear scheme, provided that the same linear solvers are applied. It is clear that our scheme would be much more efficient than the nonlinear convex-splitting schemes.

3.2.2. Second-order Scheme for the No-Slope-Model. Assume that we have found $\phi^{n}, V^{n}$ and $\phi^{n-1}, V^{n-1}$, then we compute $\phi^{n+1}, V^{n+1}$ to solve the the No-Slope-Model (2.22)-(2.23) as follows,

Step1:

$$
\frac{3 \phi^{n+1}-4 \phi^{n}+\phi^{n-1}}{2 \delta t}=-M\left(\epsilon^{2} \Delta^{2} \phi^{n+1}+\nabla \cdot\left(V^{\star} \mathbf{H}^{\star}\right)\right),
$$

with

$$
\mathbf{H}^{\star}=2 \mathbf{H}^{n}-\mathbf{H}^{n-1}, V^{\star}=2 V^{n}-V^{n-1} .
$$

Step2:

$$
\frac{3 V^{n+1}-4 V^{n}+V^{n-1}}{2 \delta t}=\mathbf{H}^{\star} \cdot \nabla \frac{3 \phi^{n+1}-4 \phi^{n}+\phi^{n-1}}{2 \delta t} .
$$

The boundary conditions are periodic or no-flux boundary conditions

$$
\left.\partial_{\mathbf{n}} \phi^{n+1}\right|_{\partial \Omega}=\left.\partial_{\mathbf{n}} \Delta \phi^{n+1}\right|_{\partial \Omega}=0 .
$$

Remark 3.11. As the first order scheme for the No-Slope-Model, the second order scheme (3.62) and (3.63) are totally decoupled. The existence and the uniqueness of the solution are obvious. Furthermore, as we can show below, the scheme is unconditionally energy stable.

THEOREM 3.8. Scheme (3.62)-(3.63) is unconditionally stable, and thus satisfies the following dis- 
crete energy dissipation law,

$$
\frac{1}{\delta t}\left(E_{B}^{n+1, n}-E_{B}^{n, n-1}\right) \leq-\frac{1}{M}\left\|\frac{3 \phi^{n+1}-4 \phi^{n}+\phi^{n-1}}{2 \delta t}\right\|^{2},
$$

where

$$
\begin{aligned}
E_{B}^{n+1, n}=\int_{\Omega} & \left\{\frac{\epsilon^{2}}{2}\left(\frac{\left(\Delta \phi^{n+1}\right)^{2}+\left(2 \Delta \phi^{n+1}-\Delta \phi^{n}\right)^{2}}{2}\right)\right. \\
& \left.-\frac{1}{2}\left(\frac{\left(V^{n+1}\right)^{2}+\left(2 V^{n+1}-V^{n}\right)^{2}-2\left(V^{n+1}-V^{n}\right)^{2}}{2}\right)+\frac{1}{2} A\right\} d \boldsymbol{x} .
\end{aligned}
$$

Proof. Taking the $L^{2}$ inner product of (3.62) with $-\frac{1}{M}\left(3 \phi^{n+1}-4 \phi^{n}+\phi^{n-1}\right)$ and performing integration by parts, we obtain

$$
\begin{aligned}
-\frac{1}{2 M \delta t}\left\|3 \phi^{n+1}-4 \phi^{n}+\phi^{n-1}\right\|^{2} & =\frac{\epsilon^{2}}{4}\left(\left\|\Delta \phi^{n+1}\right\|^{2}+\left\|2 \Delta \phi^{n+1}-\Delta \phi^{n}\right\|^{2}\right) \\
& -\frac{\epsilon^{2}}{4}\left(\left\|\Delta \phi^{n}\right\|^{2}+\left\|2 \Delta \phi^{n}-\Delta \phi^{n-1}\right\|^{2}\right) \\
& +\frac{\epsilon^{2}}{4}\left\|\Delta \phi^{n+1}-2 \Delta \phi^{n}+\Delta \phi^{n-1}\right\|^{2} \\
& -\left(V^{\star} \mathbf{H}^{\star}, \nabla\left(3 \phi^{n+1}-4 \phi^{n}+\phi^{n-1}\right)\right) .
\end{aligned}
$$

Taking the $L^{2}$ inner product of (3.63) with $2 \delta t V^{\star}$, and applying the following identity,

$$
\begin{aligned}
2(3 a-4 b+c, 2 b-c) & =\left(|a|^{2}+|2 a-b|^{2}-2|a-b|^{2}\right) \\
& -\left(|b|^{2}+|2 b-c|^{2}-2|b-c|^{2}\right)-3|a-2 b+c|^{2}
\end{aligned}
$$

we obtain

$$
\begin{aligned}
\frac{1}{2}\left(\left\|V^{n+1}\right\|^{2}+\right. & \left.\left\|2 V^{n+1}-V^{n}\right\|^{2}-2\left\|V^{n+1}-V^{n}\right\|^{2}\right)-\frac{1}{2}\left(\left\|V^{n}\right\|^{2}+\left\|2 V^{n}-V^{n-1}\right\|^{2}-2\left\|V^{n}-V^{n-1}\right\|^{2}\right) \\
& -\frac{3}{2}\left\|V^{n+1}-2 V^{n}+V^{n-1}\right\|^{2}=\left(\mathbf{H}^{\star} \cdot \nabla\left(3 \phi^{n+1}-4 \phi^{n}+\phi^{n-1}\right), V^{\star}\right)
\end{aligned}
$$

Combining (3.67) and (3.69), we obtain

$$
\begin{aligned}
\frac{\epsilon^{2}}{2}\left(\left\|\Delta \phi^{n+1}\right\|^{2}+\left\|2 \Delta \phi^{n+1}-\Delta \phi^{n}\right\|^{2}\right)-\frac{\epsilon^{2}}{2}\left(\left\|\Delta \phi^{n}\right\|^{2}+\left\|2 \Delta \phi^{n}-\Delta \phi^{n-1}\right\|^{2}\right) \\
\quad-\frac{1}{2}\left(\left\|V^{n+1}\right\|^{2}+\left\|2 V^{n+1}-V^{n}\right\|^{2}-2\left\|V^{n+1}-V^{n}\right\|^{2}\right) \\
+\frac{1}{2}\left(\left\|V^{n}\right\|^{2}+\left\|2 V^{n}-V^{n-1}\right\|^{2}-2\left\|V^{n}-V^{n-1}\right\|^{2}\right)+\frac{\epsilon^{2}}{2}\left\|\Delta \phi^{n+1}-2 \Delta \phi^{n}+\Delta \phi^{n-1}\right\|^{2} \\
+\frac{3}{2}\left\|V^{n+1}-2 V^{n}+V^{n-1}\right\|^{2}=-\frac{1}{2 M \delta t}\left\|3 \phi^{n+1}-4 \phi^{n}+\phi^{n-1}\right\|^{2}
\end{aligned}
$$

Finally we obtain the desired result (3.65) after dropping some positive terms.

Remark 3.12. From Remark 3.9, the $\frac{1}{\delta t}\left(E_{B}^{n+1, n}-E_{B}^{n, n-1}\right)$ is actually a second order approximation 
of $\frac{d}{d t} E_{B}(\phi, V)$ at $t=t^{n+1}$ if we further notice the following heuristical argument,

$$
\frac{1}{\delta t}\left(\left\|S^{n+1}-S^{n}\right\|^{2}-\left\|S^{n}-S^{n-1}\right\|^{2}\right) \cong O\left(\delta t^{2}\right) .
$$

The second order version of the Crank-Nicolson type scheme can be developed as follows,

$$
\begin{aligned}
& \frac{\phi^{n+1}-\phi^{n}}{\delta t}=-M\left(\epsilon^{2} \Delta \frac{\Delta \phi^{n+1}+\Delta \phi^{n}}{2}+\nabla \cdot\left(\frac{V^{n+1}+V^{n}}{2} \mathbf{H}^{\dagger}\right)\right), \\
& \frac{V^{n+1}-V^{n}}{\delta t}=\mathbf{H}^{\dagger} \cdot \nabla \frac{\phi^{n+1}-\phi^{n}}{\delta t},
\end{aligned}
$$

with $\mathbf{H}^{\dagger}=\frac{3}{2} \mathbf{H}^{n}-\frac{1}{2} \mathbf{H}^{n-1}$.

One can easily establish the well-posedness of the linear system and the energy stability.

Theorem 3.9. Scheme (3.71)-(3.72) is unconditionally energy stable and satisfies the following discrete energy dissipation law,

$$
\frac{1}{\delta t}\left(E_{B}^{n+1}-E_{B}^{n}\right)=-\frac{1}{M}\left\|\frac{\phi^{n+1}-\phi^{n}}{\delta t}\right\|^{2}
$$

where

$$
E_{B}^{n+1}=\int_{\Omega}\left\{\frac{\epsilon^{2}}{2}\left(\Delta \phi^{n+1}\right)^{2}-\frac{1}{2}\left(V^{n+1}\right)^{2}+\frac{1}{2} A\right\} d \boldsymbol{x} .
$$

Proof. The proof is similar to other schemes, thus the detailed proofs are left to the interested readers. We remark that the energy dissipative law is now strictly "=" instead of " $\leq$ " for other schemes.

Remark 3.13. We notice that in [3], Chen, Wang, Wang and Wise developed a second order scheme based on the convex splitting scheme to solve the the No-Slope-Model. The scheme is unconditionally stable, but nonlinear.

To the best of the authors knowledge, our second order schemes (3.62)-(3.63) and (3.71)-(3.72) are the first linear schemes with second order temporal accuracy and unconditional energy stability for the No-Slope-Model.

Remark 3.14. The local truncation error of the proposed schemes can be readily derived via Taylor series expansion assuming that the classical solutions of the PDE system have enough regularity. We omit the details here for brevity. The complete error analysis of the schemes will be pursued in a separate article.

\section{Numerical Examples.}

4.1. Spatial Discretization and Implementation. We use the central Finite Difference method to discretize the space. Let $N_{x}, N_{y}, N_{z}$ be positive integers. The domain $\Omega=\left[0, L_{x}\right] \times\left[0, L_{y}\right] \times\left[0, L_{z}\right]$, and

$$
\Omega_{h}=\left\{\left(x_{i}, y_{j}, z_{k}\right) \mid x_{i}=i h_{x}, y_{j}=j h_{y}, z_{k}=k h_{z}, 0 \leq i \leq N_{x}, 0 \leq j \leq N_{y}, 0 \leq k \leq N_{z}\right\} .
$$

We use periodic boundary conditions in all numerical simulations. Let $x_{-1}=-h_{x}, y_{-1}=-h_{y}$, 


\begin{tabular}{c||c|c|c|c}
\hline$\delta t$ & $L^{2}-$ Error of A-LS1 & Order & $L^{2}-$ Error of A-LS2 & Order \\
\hline \hline $1 \times 10^{-2}$ & $7.8438 \mathrm{e}-02$ & & $7.7811 \mathrm{e}-02$ & \\
\hline $5 \times 10^{-3}$ & $4.1614 \mathrm{e}-02$ & 0.914 & $2.2754 \mathrm{e}-02$ & 1.774 \\
\hline $2.5 \times 10^{-3}$ & $2.1628 \mathrm{e}-02$ & 0.944 & $6.6622 \mathrm{e}-03$ & 1.772 \\
\hline $1.25 \times 10^{-3}$ & $1.1012 \mathrm{e}-02$ & 0.974 & $1.8850 \mathrm{e}-03$ & 1.821 \\
\hline $6.25 \times 10^{-4}$ & $5.5219 \mathrm{e}-03$ & 0.996 & $5.1090 \mathrm{e}-04$ & 1.883 \\
\hline $3.125 \times 10^{-4}$ & $2.7304 \mathrm{e}-03$ & 1.016 & $1.3391 \mathrm{e}-04$ & 1.932 \\
\hline $1.5625 \times 10^{-4}$ & $1.3235 \mathrm{e}-03$ & 1.045 & $3.4296 \mathrm{e}-05$ & 1.965 \\
\hline $7.8125 \times 10^{-5}$ & $6.1729 \mathrm{e}-04$ & 1.100 & $8.5962 \mathrm{e}-06$ & 1.996 \\
\hline
\end{tabular}

TABLE 4.1

Cauchy convergence rate test for schemes A-LS1, A-LS2, respectively. The errors are measured in the $L^{2}$ norm with $\delta t=\frac{10^{-2}}{2^{k}}, k=0,1, \cdots, 7$ with default order parameters (4.5).

$z_{-1}=-h_{z}$. A grid function is called periodic if

$$
\begin{array}{ll}
\text { x periodic } & f_{-1, j, k}=f_{N_{x}-1, j, k}, f_{0, j, k}=F_{N_{x}, j, k} . \\
\text { y periodic } & f_{i,-1, k}=f_{i, N_{y}-1, k}, f_{i, 0, k}=F_{i, N_{y}, k} . \\
\text { z periodic } & f_{i, j,-1}=f_{i, j, N_{z}-1}, f_{i, j, 0}=F_{i, j, N_{z}} .
\end{array}
$$

In order to derive the algorithm conveniently, we introduce the following discrete difference operators

$$
\begin{array}{lll}
\delta_{x}^{+}=\frac{f_{i+1, j, k}-f_{i, j, k}}{h_{x}}, & \delta_{x}^{-}=\frac{f_{i, j, k}-f_{i-1, j, k}}{h_{x}}, & \delta_{\bar{x}}=\frac{\delta_{x}^{+}+\delta_{x}^{-}}{2} f_{i, j, k}, \\
\delta_{y}^{+}=\frac{f_{i, j+1, k}-f_{i, j, k}}{h_{y}}, & \delta_{y}^{-}=\frac{f_{i, j, k}-f_{i, j-1, k}}{h_{y}}, & \delta_{\bar{y}}=\frac{\delta_{y}^{+}+\delta_{y}^{-}}{2} f_{i, j, k}, \\
\delta_{z}^{+}=\frac{f_{i, j, k+1}-f_{i, j, k}}{h_{z}}, & \delta_{z}^{-}=\frac{f_{i, j, k}-f_{i, j, k-1}}{h_{z}}, & \delta_{\bar{z}}=\frac{\delta_{z}^{+}+\delta_{z}^{-}}{2} f_{i, j, k},
\end{array}
$$

and

$$
\nabla_{h}^{+}=\left(\begin{array}{c}
\delta_{x}^{+} \\
\delta_{y}^{+} \\
\delta_{z}^{+}
\end{array}\right), \quad \nabla_{h}^{-}=\left(\begin{array}{c}
\delta_{x}^{-} \\
\delta_{y}^{-} \\
\delta_{z}^{-}
\end{array}\right), \quad \bar{\nabla}_{h}=\frac{\nabla_{h}^{+}+\nabla_{h}^{-}}{2}, \quad \Delta_{h}=\delta_{x}^{+} \delta_{x}^{-}+\delta_{y}^{+} \delta_{y}^{-}+\delta_{z}^{+} \delta_{z}^{-} .
$$

By replacing the continuous operator $\Delta, \nabla$ with the discrete operator $\Delta_{h}$ and $\bar{\nabla}_{h}$, one can show that the discrete energy laws of the fully discrete schemes still hold in the same way as in the semi discrete schemes.

4.2. Numerical Results. We next present various numerical examples to validate the theoretical results derived in the previous section and demonstrate the efficiency, the stability and the accuracy 


\begin{tabular}{c||c|c|c|c}
\hline$\delta t$ & $L^{2}-$ Error of B-LS1 & Order & $L^{2}-$ Error of B-LS2 & Order \\
\hline \hline $1 \times 10^{-2}$ & $1.5195 \mathrm{e}-02$ & & $1.5192 \mathrm{e}-02$ & \\
\hline $5 \times 10^{-3}$ & $5.5088 \mathrm{e}-03$ & 1.463 & $5.7043 \mathrm{e}-03$ & 1.413 \\
\hline $2.5 \times 10^{-3}$ & $1.8934 \mathrm{e}-03$ & 1.540 & $1.6942 \mathrm{e}-03$ & 1.752 \\
\hline $1.25 \times 10^{-3}$ & $6.4687 \mathrm{e}-04$ & 1.549 & $4.5984 \mathrm{e}-04$ & 1.881 \\
\hline $6.25 \times 10^{-4}$ & $2.3869 \mathrm{e}-04$ & 1.438 & $1.2009 \mathrm{e}-04$ & 1.937 \\
\hline $3.125 \times 10^{-4}$ & $9.9064 \mathrm{e}-05$ & 1.269 & $3.0697 \mathrm{e}-05$ & 1.968 \\
\hline $1.5625 \times 10^{-4}$ & $4.4352 \mathrm{e}-05$ & 1.159 & $7.7355 \mathrm{e}-06$ & 1.989 \\
\hline $7.8125 \times 10^{-5}$ & $2.0011 \mathrm{e}-05$ & 1.148 & $1.9194 \mathrm{e}-06$ & 2.011 \\
\hline
\end{tabular}

TABLE 4.2

Cauchy convergence rate test for schemes B-LS1, B-LS2, respectively. The errors are measured in the $L^{2}$ norm with $\delta t=\frac{10^{-2}}{2^{k}}, k=0,1, \cdots, 7$ with default order parameters $(4.5)$.

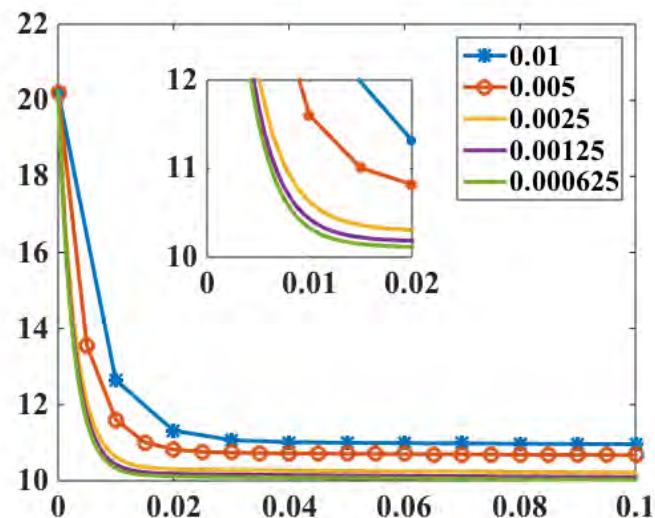

(a) the Slope-Model.

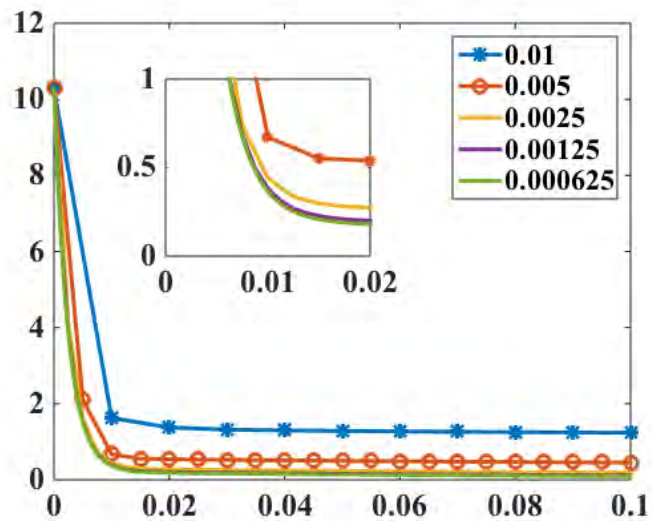

(b) the No-Slope-Model.

FIG. 4.1. Time evolution of the free energy functional of the Slope-Model and the No-Slope-Model for five different time step sizes of $\delta t=0.000625,0.00125,0.0025,0.005$, and 0.01 for example 4.2.1 with the initial profile (4.6) and the default parameters (4.5). The small inset figure shows the small differences in the energy evolution for the considered time step sizes of $\delta t=0.00125$ and 0.000625 .

of the new numerical schemes. In all examples, we use periodic boundary conditions in the square domain $[0, L]^{2}$. If not explicitly specified, the default values of parameters are given as follows, which is consistent with the numerical examples performed in the literatures [17,27, 29],

$$
\epsilon^{2}=0.1, M=1 \text {. }
$$




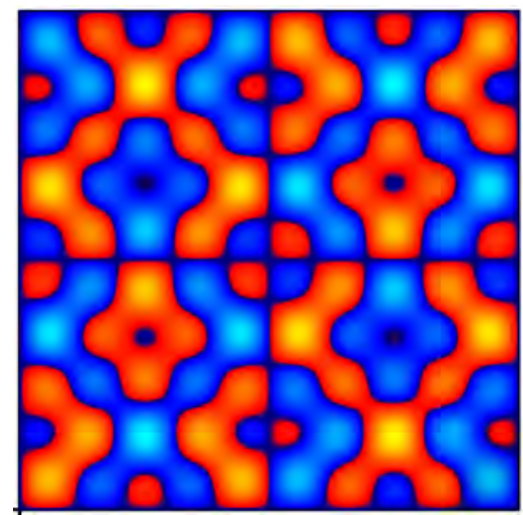

(a) $t=0$.

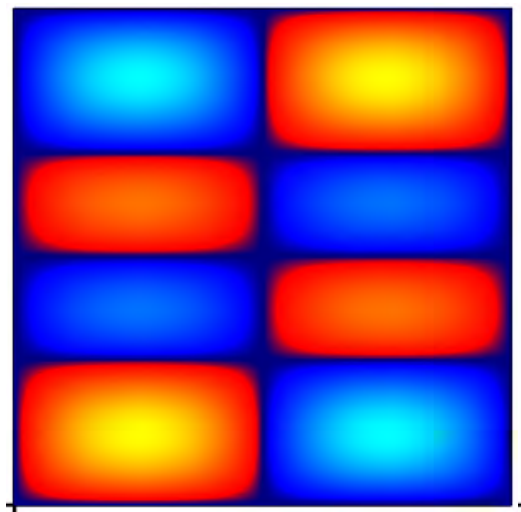

(d) $t=5.5$.

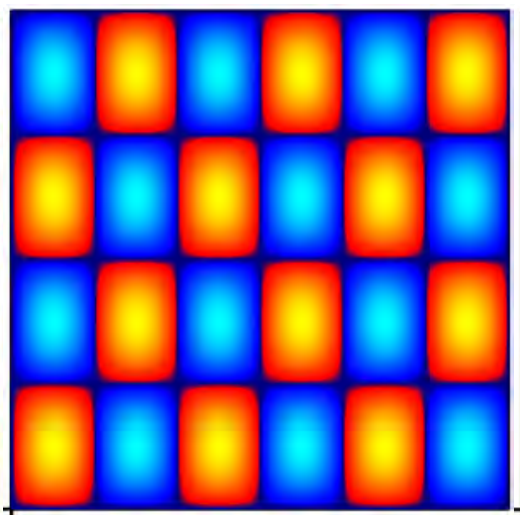

(b) $t=0.05$.

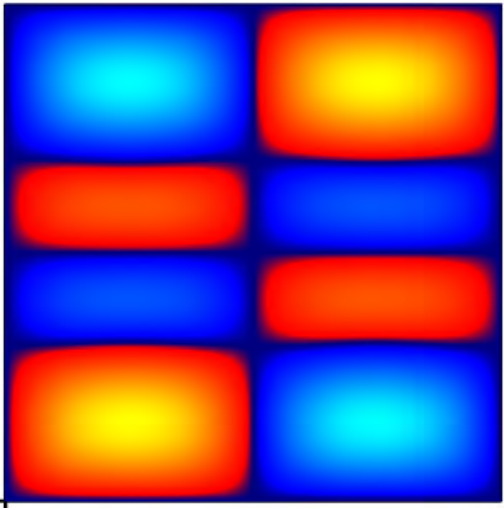

(e) $t=8$.

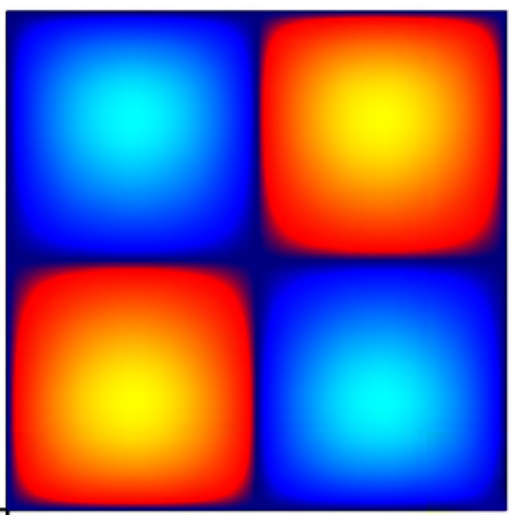

(c) $t=2.5$.

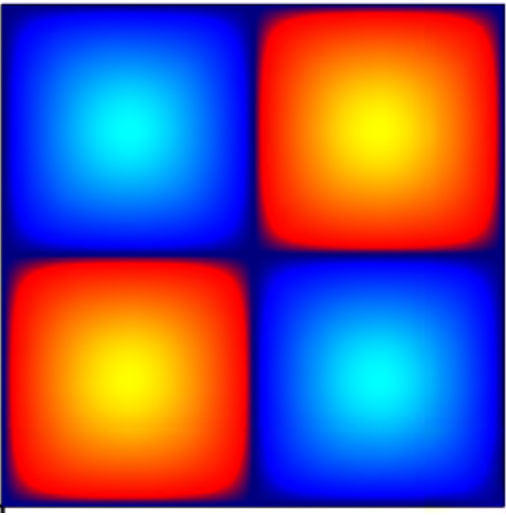

(f) $t=30$.

FIG. 4.2. The isolines of numerical solutions of the height function $\phi$ for the Slope-Model of example 4.2.1 using the second order scheme A-LS2. Snapshots are taken at $t=0,0.05,2.5,5.5,8,30$, respectively.

4.2.1. Convergence rate test . We first test convergence rates of the four new schemes through a particular example that had been well studied for the case of the Slope-Model in $[17,29]$. We denote the first order linear scheme (3.1)-(3.2) for the Slope-Model by A-LS1; the second order linear scheme (3.44)(3.45) for the Slope-Model by A-LS2; the first order linear scheme (3.26)-(3.28) for the No-Slope-Model by B-LS1; and the second order linear scheme of BDF2 version (3.62)-(3.63) for the No-Slope-Model by B-LS2. Limitations of space prevent us to discuss the numerical tests of the Crank-Nicolson schemes, thus we leave that to the interested readers.

The following initial condition

$$
\phi(t=0)=0.1(\sin 3 x \sin 2 y+\sin 5 x \sin 5 y)
$$

is used in all tests. The computational domain is $[0,2 \pi]^{2}$ and $128 \times 128$ grid points are used to discretize the space. To obtain the deviation of the height function, we define the roughness measure function $W(t)$ as follows (cf. [17,27,29]), $W(t)=\sqrt{\frac{1}{|\Omega|} \int_{\Omega}(\phi(\boldsymbol{x}, t)-\bar{\phi}(\boldsymbol{x}, t))^{2} d \boldsymbol{x}}$, where $\bar{\phi}(t)=\frac{1}{\Omega} \int_{\Omega} \phi(\boldsymbol{x}, t) d \boldsymbol{x}$ is the average. In the context of the Finite Difference Method, the discrete version of the roughness is 


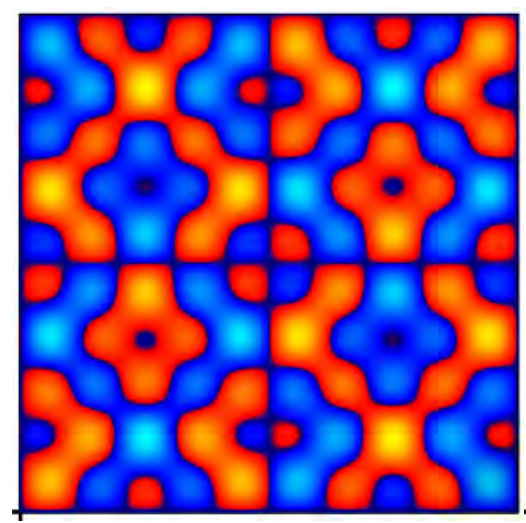

(a) $t=0$.

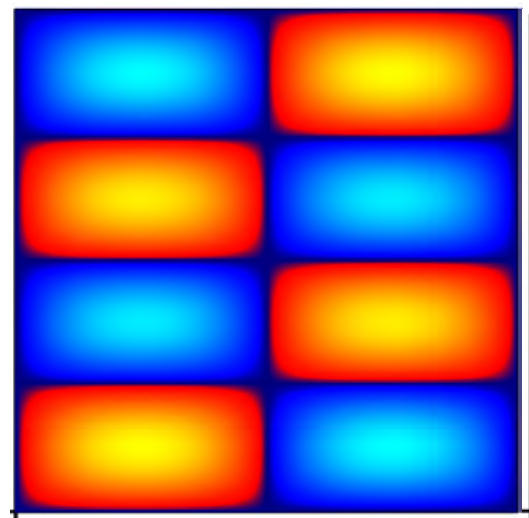

(d) $t=5.5$.

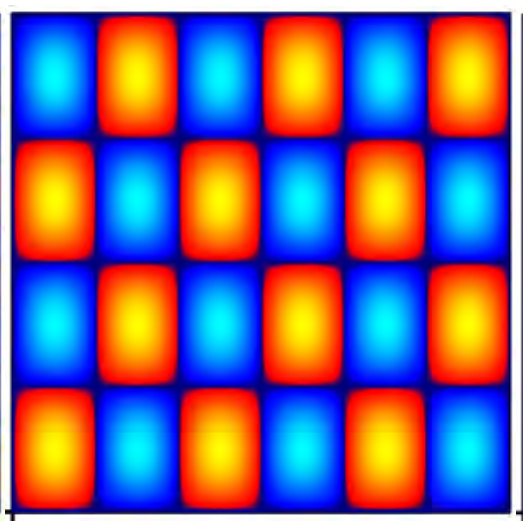

(b) $t=0.05$.

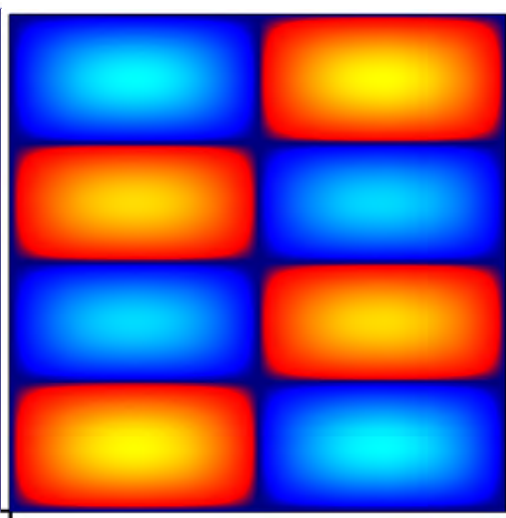

(e) $t=8$.

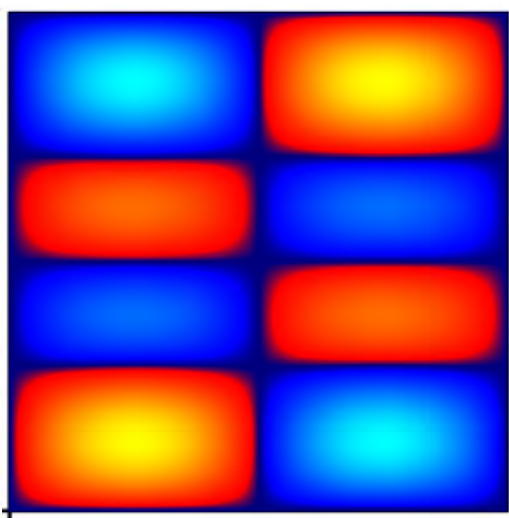

(c) $t=2.5$.

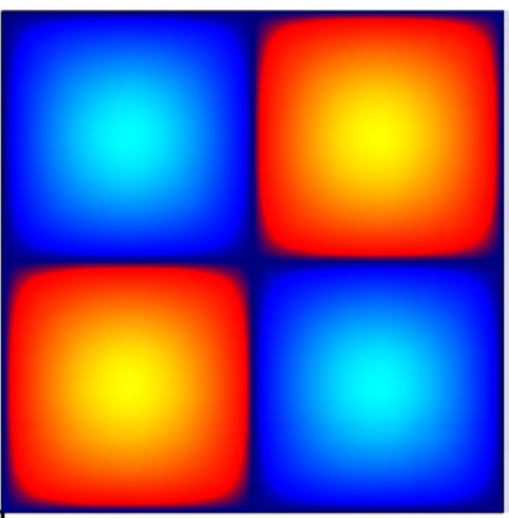

(f) $t=30$.

FIG. 4.3. The isolines of numerical solutions of the height function $\phi$ for the No-Slope-Model of example 4.2.1 using the second order scheme B-LS2. The time step is $\delta t=10^{-3}$. Snapshots are taken at $t=0,0.05,2.5,5.5,8,30$, respectively.

$W\left(t_{n}\right)=\sqrt{\frac{h_{x} h_{y}}{L_{x} L_{y}} \sum_{i=1}^{N x} \sum_{j=1}^{N y}\left(\phi_{i j}^{n}-\bar{\phi}^{n}\right)^{2}}$.

We first perform the mesh refinement test in time and choose the numerical solution using the second order schemes A-LS2 and B-LS2 with the smallest time step size of $\delta t=1.0 \times 10^{-5}$ as the benchmark solution (approximate exact solution) for computing errors, respectively. For the Slope-Model, in Table 4.1, we give the $L^{2}$ errors between the numerical solution and the benchmark solution at $t=1.0$ with different time step sizes using schemes A-LS1 and A-LS2. For the No-Slope-Model, in Table 4.2, we give the $L^{2}$ errors between the numerical solution and the benchmark solution at $t=1.0$ with different time step sizes using schemes B-LS1 and B-LS2 as well. We observe that all four schemes yield good convergence rates that asymptotically (at least) match their expected orders in time. Moreover, the second order schemes give much better accuracy than the first order schemes when using the same time step. Thus, we adopt the second order schemes A-LS2 and B-LS2 in order to obtain better accuracy.

We emphasize that any time step $\delta t$ is allowable for the computations from the stability concern since all proposed numerical schemes are unconditionally energy stable. Thus one needs to discover the range of the critical time step size to obtain good accuracy and to consume as low computational 

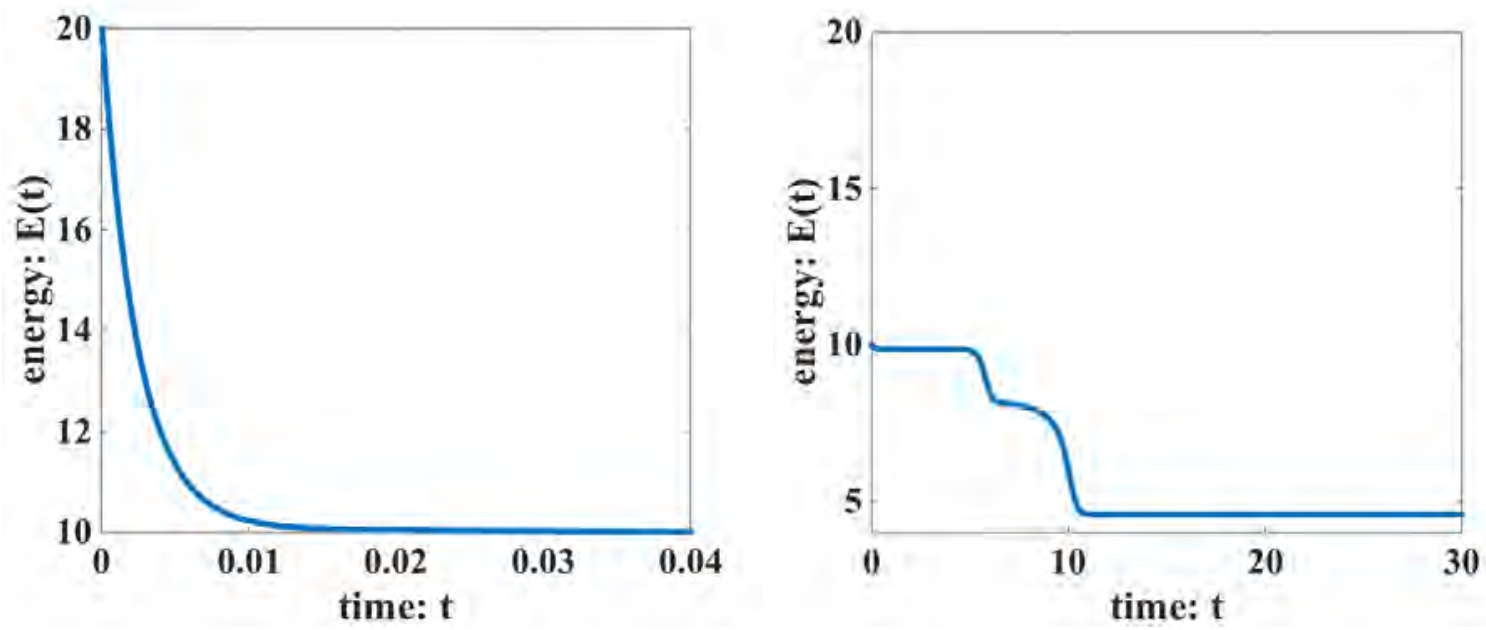

(a) Energy evolution for the Slope-Model when $t \in(0,0.04)$ and $t \in(0,30)$.
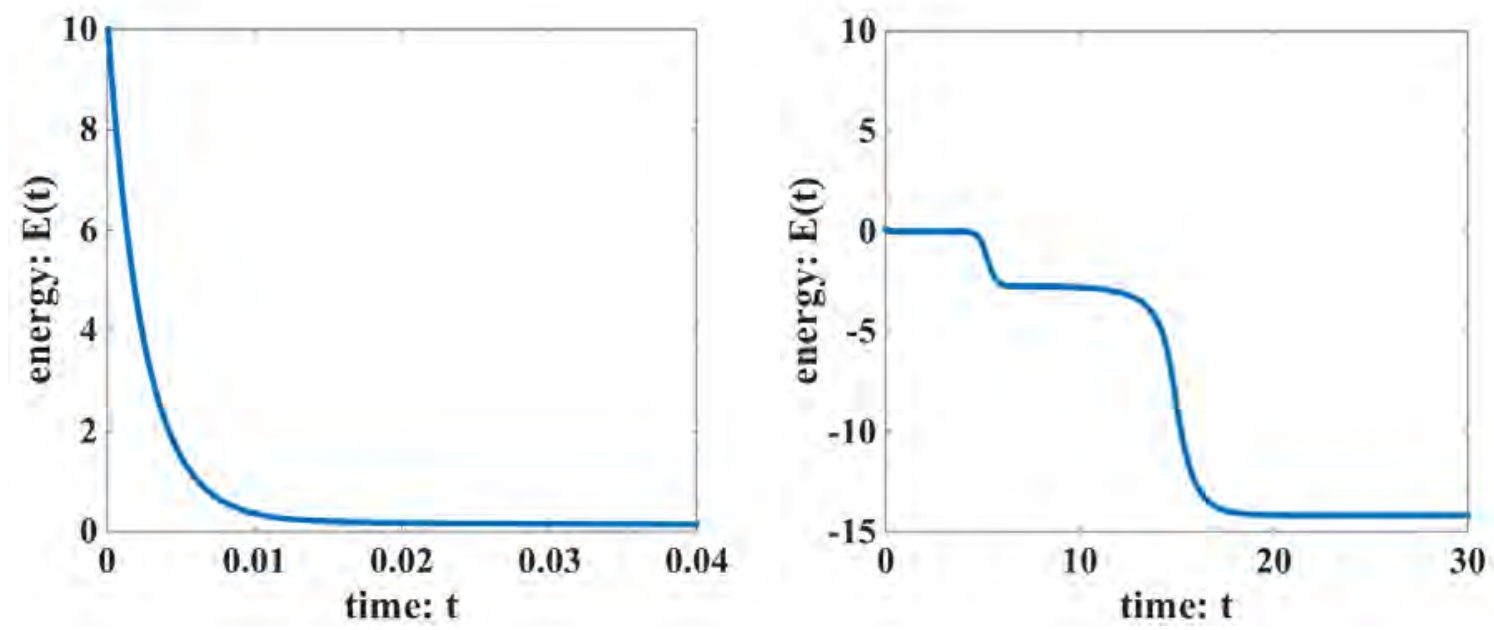

(b) Energy evolution for the No-Slope-Model when $t \in(0,0.04)$ and $t \in(0,30)$.

FIG. 4.4. Time evolution of the energy for the Slope-Model using A-LS2 and the No-Slope-Model using B-LS2, respectively. The time step is set at $\delta t=10^{-3}$ for both models.

cost as possible for the particular initial value and parameters. This critical time step can be roughly estimated from the energy plots with the time step. In Fig. 4.1, we compare the time evolution of the free energy functional for five different time step sizes until $T=0.1$ for the Slope-Model and the No-Slope-Model using the second order schemes A-LS2 and B-LS2, respectively. All five energy curves show decays for all time step sizes, which confirm that our algorithms are unconditionally stable for any time step. Moreover, for smaller time steps of $\delta t=0.00125$ and 0.000625 , the two energy curves coincide. For larger time steps of $\delta t=0.0025,0.005$ and 0.01 , the energy curves deviate away from others. This means the time step size has to be smaller than 0.0025 at least, in order to get reasonably good accuracy. Therefore, for the simulations below, we use $\delta t=0.001$ to study growth dynamics of the height function $\phi$. 

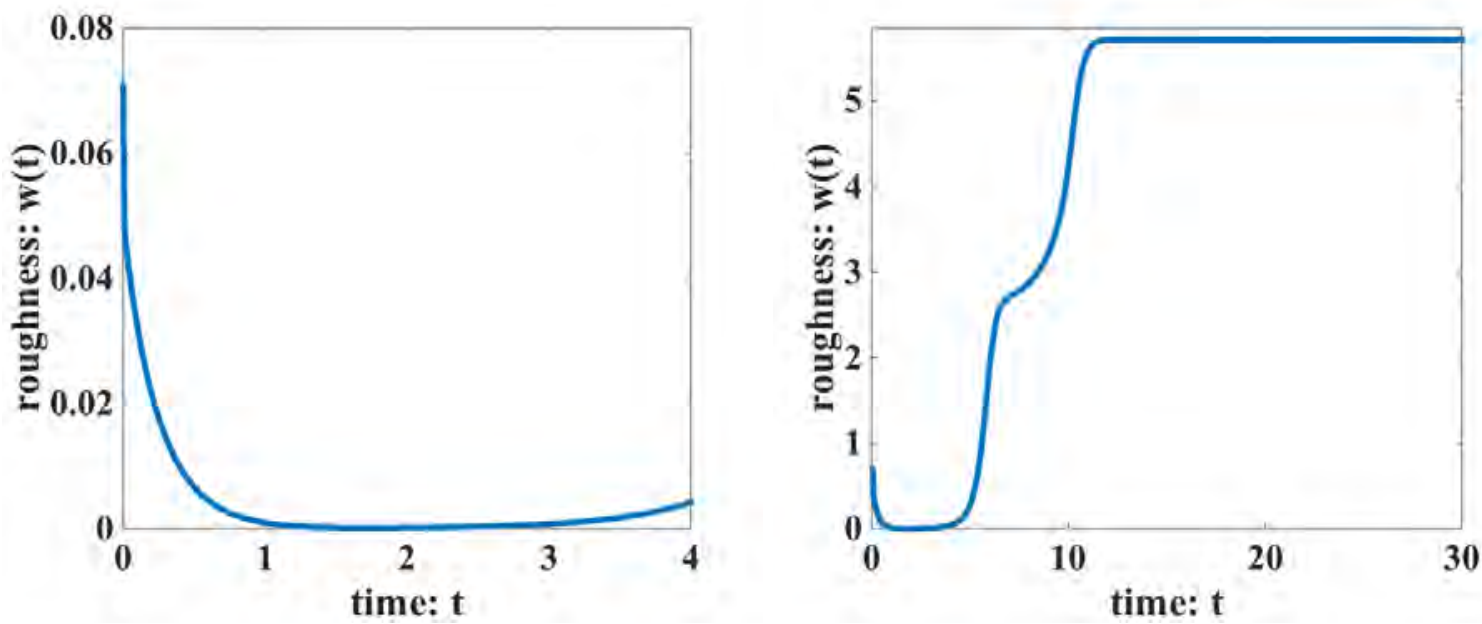

(a) Roughness evolution for the Slope-Model when $t \in(0,0.04)$ and $t \in(0,30)$.
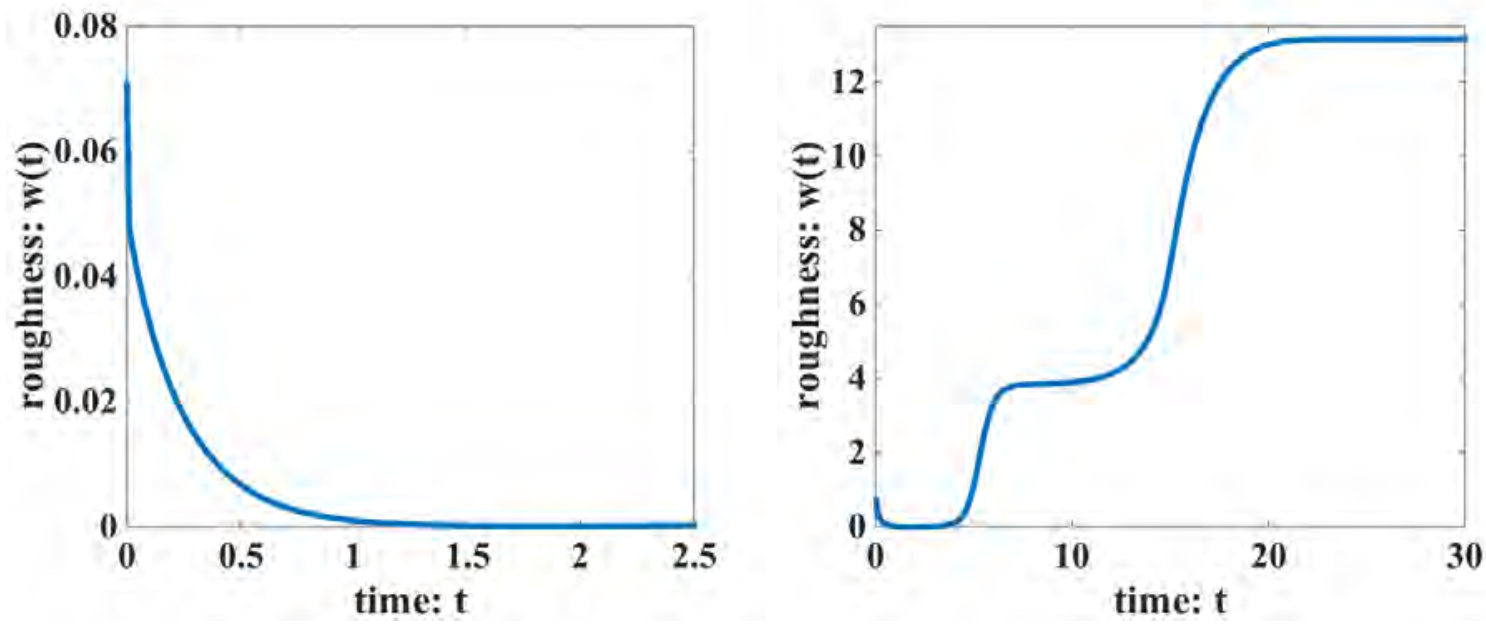

(b) Roughness evolution for the No-Slope-Model when $t \in(0,0.04)$ and $t \in(0,30)$.

FIG. 4.5. Time evolution of the roughness for the Slope-Model using A-LS2 and the No-Slope-Model using B-LS2, respectively. The time step is set at $\delta t=10^{-3}$ for both models.

In Fig. 4.2 and 4.3, we show the isolines of numerical solutions of $\phi$ up to the steady state $(t=$ 30) using the second order schemes A-LS2 and B-LS2 for the Slope-Model and the No-Slope-Model, respectively. These two models predict similar patterns with some slight differences. We plot the evolution of energy curves for the Slope-Model and the No-Slope-Model in Fig. 4.4, and the roughness for the Slope-Model and the No-Slope-Model in Fig. 4.5, respectively. We observe that initially the energy and the roughness decay rapidly. After a relatively long period of time, the roughness starts to grow. Overall, the numerical solutions present the quantitatively identical features when compared with those results obtained in [17]. Unfortunately, we have no way to compare the effectiveness with the numerical algorithms presented in [17] since their corresponding time steps were not specified. 


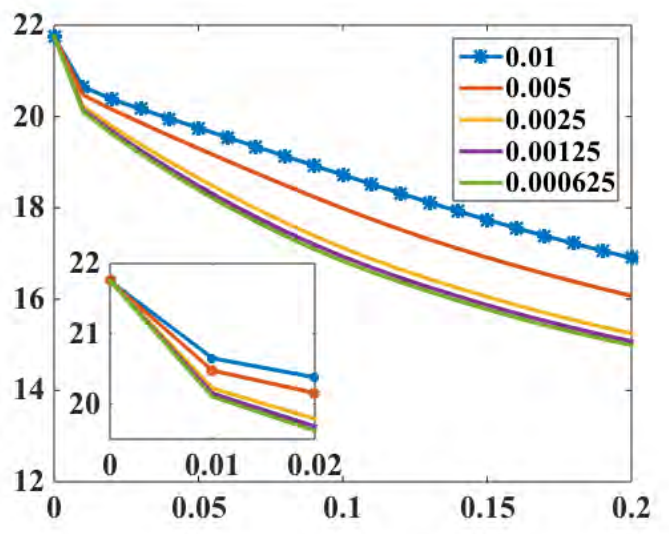

(a) the Slope-Model.

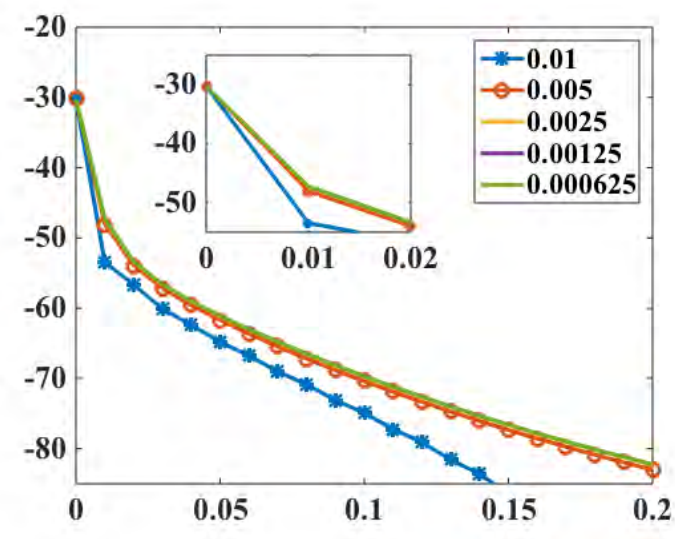

(b) the No-Slope-Model.

FIG. 4.6. Time evolution of the energy functional of the Slope-Model and the No-Slope-Model for five different time step sizes, $\delta t=0.000625,0.00125,0.0025,0.005$, and 0.01 for example 4.2.2 with the initial profile of the random number in [-0.001, 0.001] and default parameters (4.5). The small inset figure in subfigure (a) shows the small difference in the energy evolution for the considered time steps of $\delta t=0.000625$ and 0.00125 , the small inset figure in the subfigure (b) shows the small difference in the energy evolution for the considered time steps of $\delta t=0.005,0.0025,0.00125$ and 0.000625 , respectively.

4.2.2. Coarsening dynamics. In this example, we perform numerical simulations of coarsening dynamics in $2 \mathrm{D}$. The initial condition is a random state by assigning a random number varying from -0.001 to 0.001 to each grid point. We use second order schemes A-LS2 and B-LS2 for better accuracy. The simulations are carried out in the domain $[0, L]^{2}$ with $L=12.8$, and with the periodic boundary conditions in space. We use $512^{2}$ grid points so that the errors from the spatial discretization is negligibly small compared with the time discretization error.

We still need to discover the range of the critical time step size to obtain good accuracy and consume as low computational cost as possible for this particular initial value. In Fig. 4.6, we compare the evolution of the free energy functional for five different time step sizes until $T=0.1$ for the Slope-Model and the No-Slope-Model using the second order schemes A-LS2 and B-LS2, respectively. All five energy curves show decays for all time step sizes, which confirm that our algorithms are unconditionally stable for any time step. For the Slope-Model, the two energy curves using smaller time steps of $\delta t=0.00125$ and 0.000625 coincide. For larger time steps of $\delta t=0.0025,0.005$ and 0.01 , the energy curves deviate considerable away from others. This means the time step size has to be smaller than 0.0025 at least in order to get good accuracy. For the No-Slope-Model, things are much better, where the four energy curves using $\delta t=0.005,0.0025,0.00125,0.000625$ all coincide. The energy curve using the largest time step of $\delta t=0.01$, deviate considerable away from others. Therefore, for the simulations below, we use $\delta t=0.001$ to study growth dynamics of the height function $\phi$.

In Fig. 4.7 and Fig. 4.8, we show snapshots of numerical solutions of the height function $\phi$ and its Laplacian $\Delta \phi$ at different time snapshots for the Slope-Model and the No-Slope-Model, respectively. We observe the growth of the epitaxial film where the pyramid/anti-pyramid shapes of hills and valleys. As predicted by [15], for the Slope-Model, the energy is expected to decrease approximately like $t^{1 / 3}$, which is shown in Fig. 4.9 (a). The growth rate of the roughness function $t^{\frac{1}{3}}$ is also predicted in $[15,17,18]$, which is also observed in Fig. 4.10 (a). For the No-Slope-Model, shown in Fig. 4.9 (b), the energy decays rather rapidly like $-\log _{10}(t)$ as predicted in [17]. The growth rate of the roughness is $t^{0.49}$ is shown in Fig. 4.10 (b). All of these numerical solutions present similar and consistent features to those 


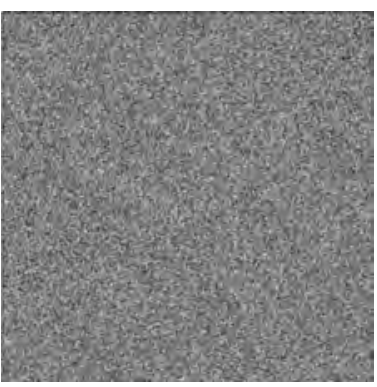

(a) $\mathrm{t}=0$.
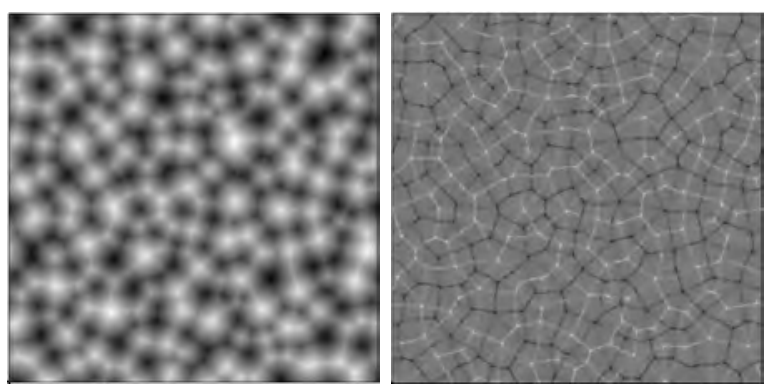

(c) $\mathrm{t}=10$.
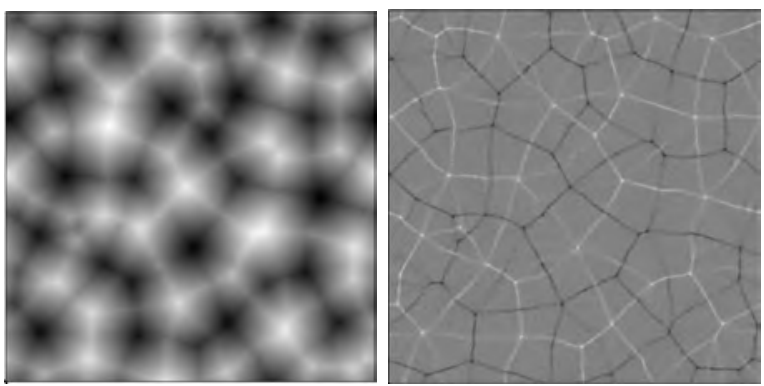

(e) $\mathrm{t}=100$

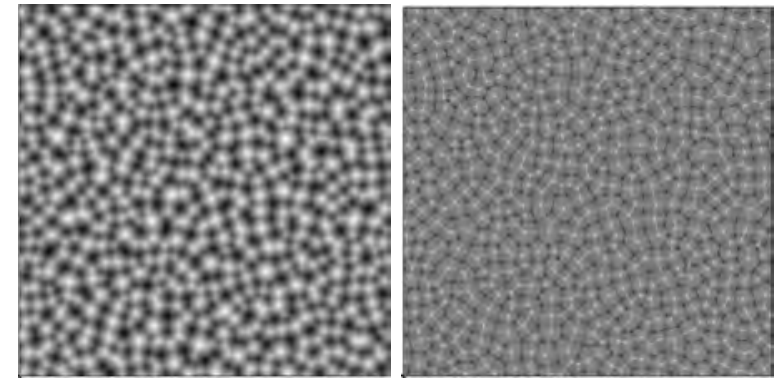

(b) $\mathrm{t}=1$.
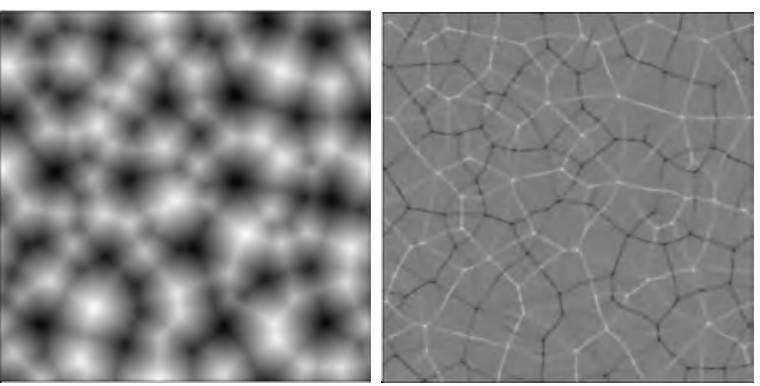

(d) $\mathrm{t}=50$.
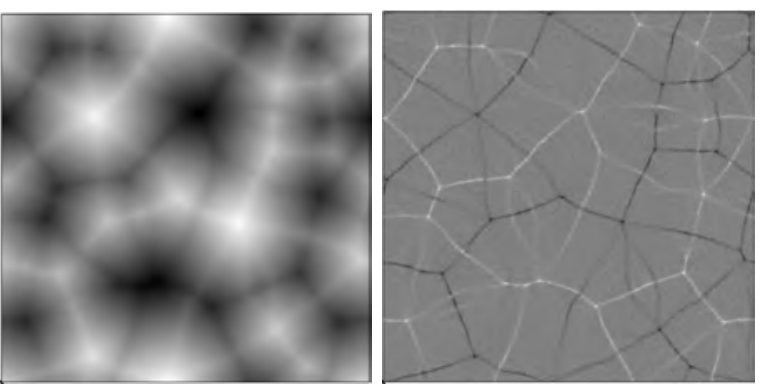

(f) $\mathrm{t}=500$.

FIG. 4.7. The isolines of the numerical solutions of the height function $\phi$ and its Laplacian $\Delta \phi$ for the Slope-Model of example 4.2.2 using schemes A-LS2 with the random initial condition and time step $\delta t=10^{-3}$. For each subfigure, the left is $\phi$ and the right is $\Delta \phi$. Snapshots are taken at $t=0,1,10,50,100,500$, respectively. Here we choose $\varepsilon=0.03$.

obtained in [27] using the convex splitting approach.

We finally perform numerical simulations of the coarsening dynamics in $3 \mathrm{D}$ with the same random state by assigning a random number from -0.001 to 0.001 to each grid point as the initial condition. Using the second order schemes A-LS2 and B-LS2, and the periodic boundary conditions, the simulations are carried out in the domain $[0, L]^{3}$ with $L=3.14$. We use $128 \times 128 \times 128$ grid points to discretize the space and the time step is $\delta t=10^{-3}$. We show three isosurfaces for $\phi=-0.025,0$ and 0.025 with three colors (blue, green and red) in Fig. 4.11 and Fig. 4.12 for the Slope-Model and the No-Slope-Model, respectively.

5. Concluding Remarks. In this paper, we have developed a set of effective, efficient, semidiscrete in time, first and second order schemes for solving the continuum MBE model using the novel 


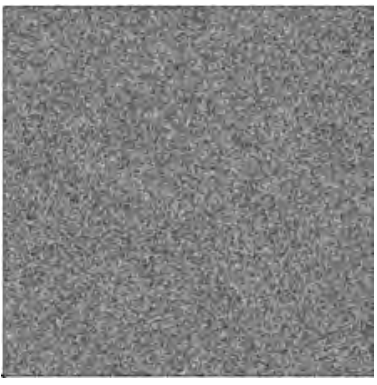

(a) $\mathrm{t}=0$.

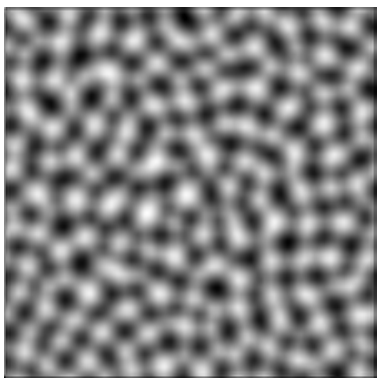

(c) $\mathrm{t}=10$.

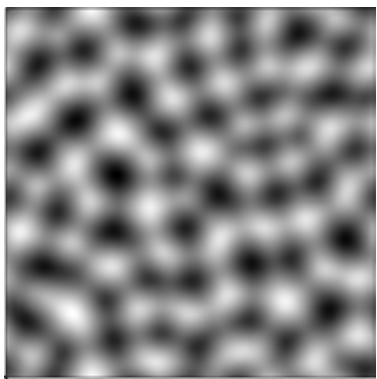

(e) $\mathrm{t}=100$.

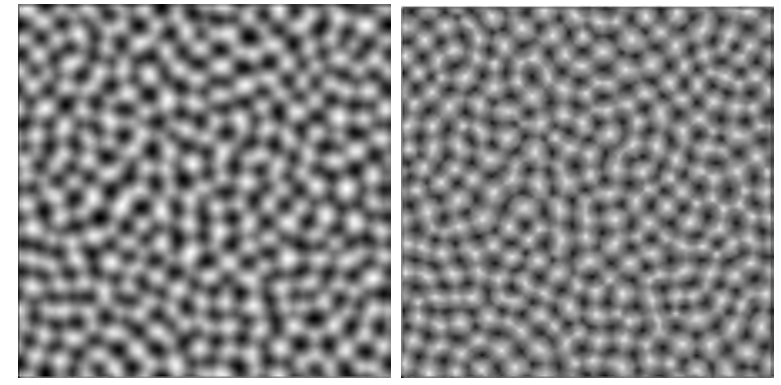

(b) $\mathrm{t}=1$.

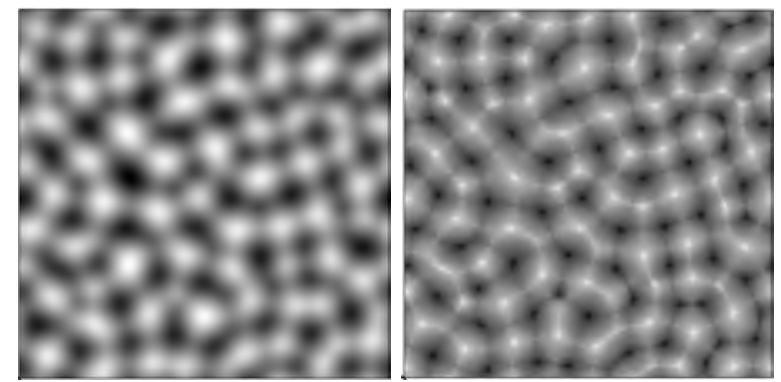

(d) $\mathrm{t}=50$.
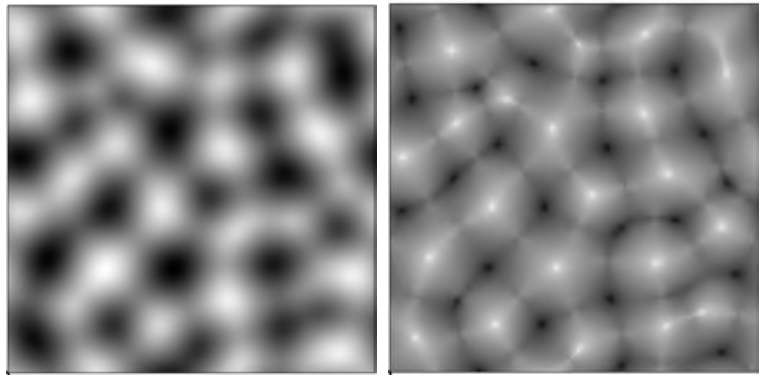

(f) $\mathrm{t}=500$.

FIG. 4.8. The isolines of numerical solutions of the height function $\phi$ and its Laplacian $\Delta \phi$ for the No-Slope-Model of example 4.2.2 using schemes B-LS2 with the random initial condition and time step $\delta t=10^{-3}$. For each subfigure, the left is $\phi$ and the right is $\Delta \phi$. Snapshots are taken at $t=0,1,10,50,100,500$, respectively. Here we choose $\varepsilon=0.03$.

IEQ approach. The proposed schemes possess unconditional energy stability without resorting to convex splitting nor adding stabilizers. Specifically, the new schemes are linear, accurate and unconditional energy stable. Furthermore, for the Slope-Model, the linear operator of the systems are symmetric positive definite, thus one can apply any Krylov subspace methods as pre-conditioners to solve them efficiently. For the No-Slope-Model, the proposed schemes are linear and decoupled. We note that these schemes can also serve as a building block to design accurate and stable linear schemes for a class of gradient flow problems with high degree of nonlinearity (cf. the schemes for the classical Cahn-Hilliard, Allen-Cahn model and phase field crystal model in [11,13,32]). Although we consider only semidiscretized schemes in time in this paper, the results here can be carried over to any consistent finitedimensional Galerkin type approximations since the proofs are all based on a variational formulation 


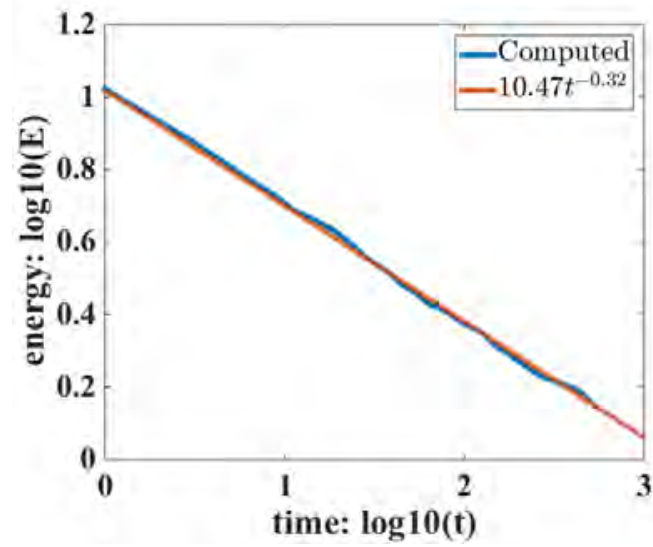

(a) the Slope-Model.

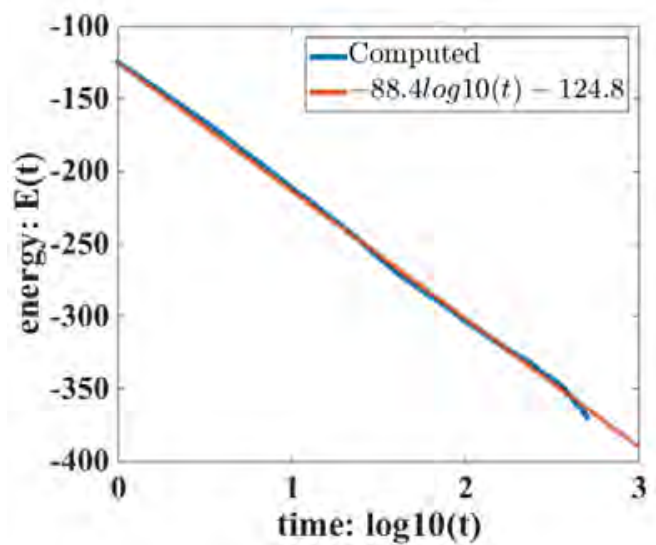

(b) the No-Slope-Model.

FIG. 4.9. The log-log, and semi-log plots of the time evolution of the energy for the Slope-Model and the No-Slope-Model, respectively. For the Slope-Model, the energy decreases like $t^{-1 / 3}$ while for the No-Slope-Model, the energy decreases like $\log _{10}(t)$, until saturation. The blue lines represent the energy plot obtained by the simulations, while the straight red lines are obtained by least square approximations to the energy data. The least squares fit is only up to about time $t=500$.

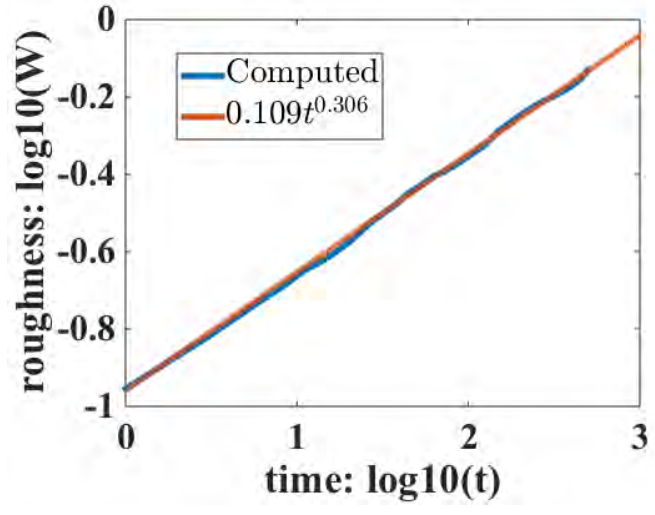

(a) the Slope-Model.

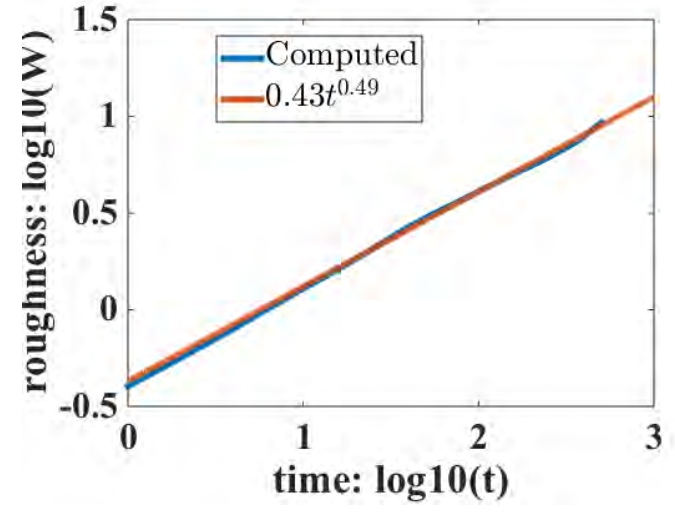

(b) the No-Slope-Model.

FiG. 4.10. The log-log plots of the temporal evolution the roughness for the Slope-Model and the No-Slope-Model, respectively. For the Slope-Model, the roughness increases like $t^{1 / 3}$ and for the No-Slope-Model, the roughness increases like $t^{1 / 2}$ until saturation. The blue lines represent the energy plot obtained by simulations, while the straight red lines are obtained by least-square approximations to the energy data. The least-square fit is only up to about time $t=500$.

with all test functions in the same space as the space of the trial functions.

\section{REFERENCES}

[1] R. E. Caflisch, M. F. Gyure, B. Merriman, S. Osher, C. Ratsch, D. D. Vvedensky, and J. J. Zinck. Island dynamics and the level set method for epitaxial growth. Appl. Math. Lett., 12:13-22, 1999.

[2] W. Chen, S. Conde, C. Wang, X. Wang, and S. Wise. A linear energy stable scheme for a thin film model without slope selection. Journal of Scientific Computing, 52:546-562, 2012. 


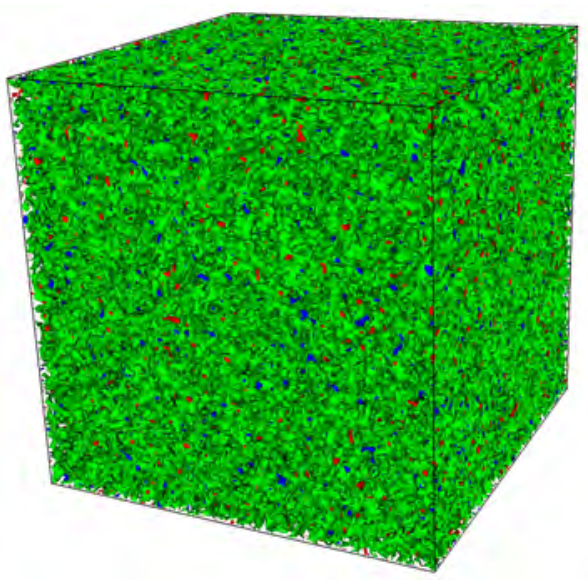

(a) $t=0$.

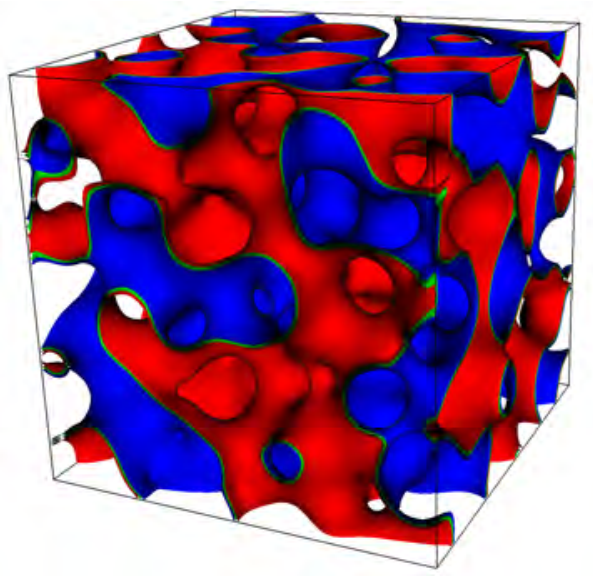

(c) $t=2$.

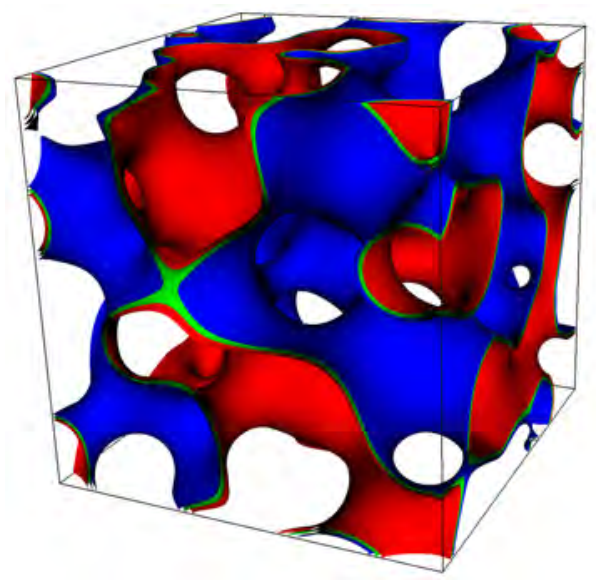

(e) $t=10$.

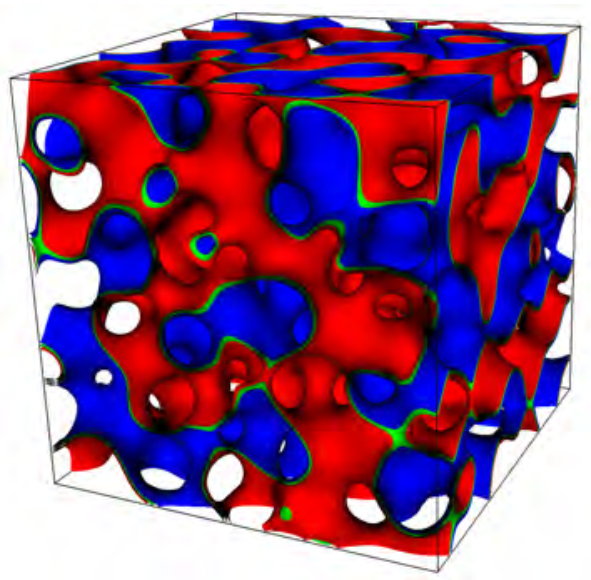

(b) $t=1$.

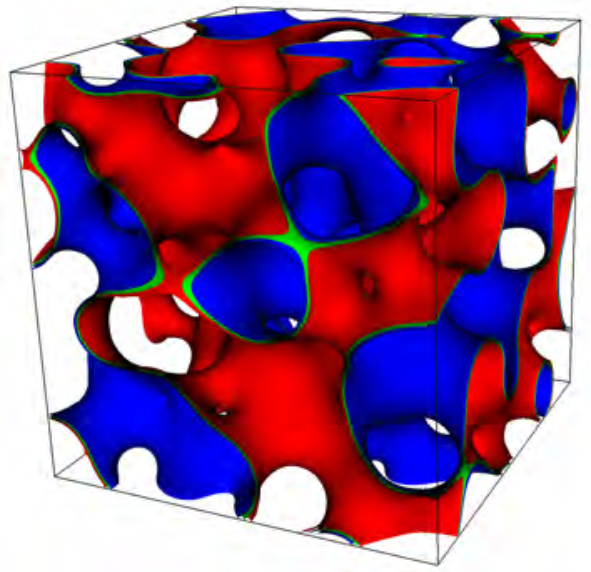

(d) $t=5$.

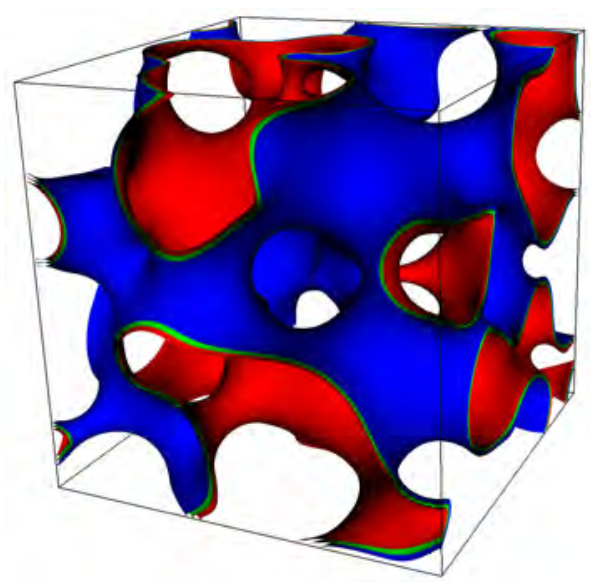

(f) $t=15$.

FIG. 4.11. The three isosurfaces of numerical solutions of the height function $\phi=-0.025,0$ and 0.025 for the Slope-Model of the 3D coarsening dynamics example using the second order scheme A-LS2, with the random initial condition and time step $\delta t=10^{-3}$. Snapshots are taken at $t=0,1,2,5,10,15$, respectively. Here we choose $\varepsilon=0.03$ 


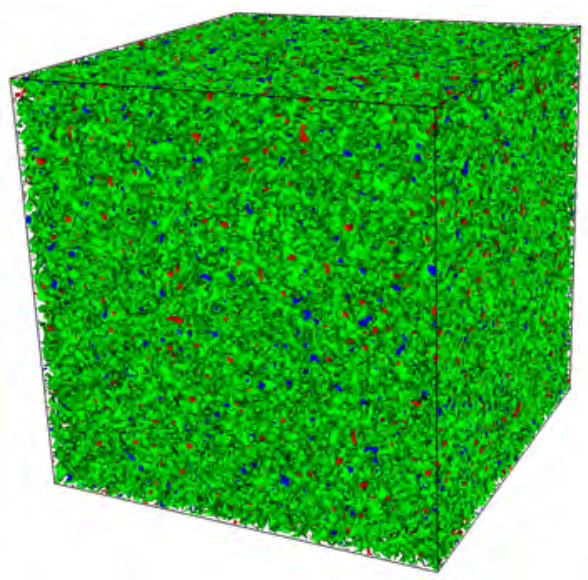

(a) $t=0$.

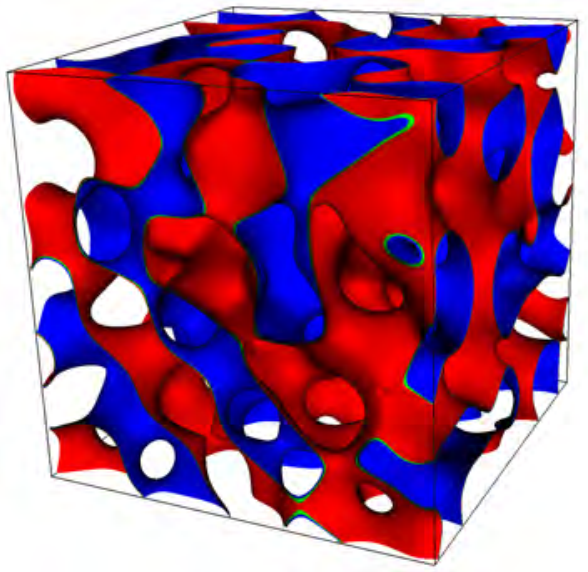

(c) $t=2$.

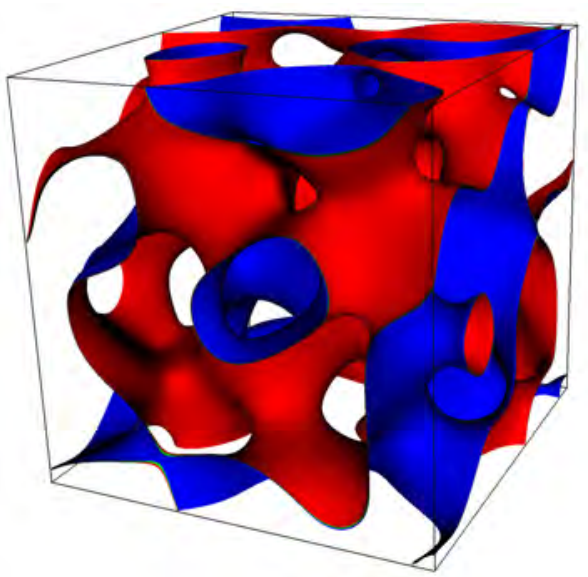

(e) $t=10$.

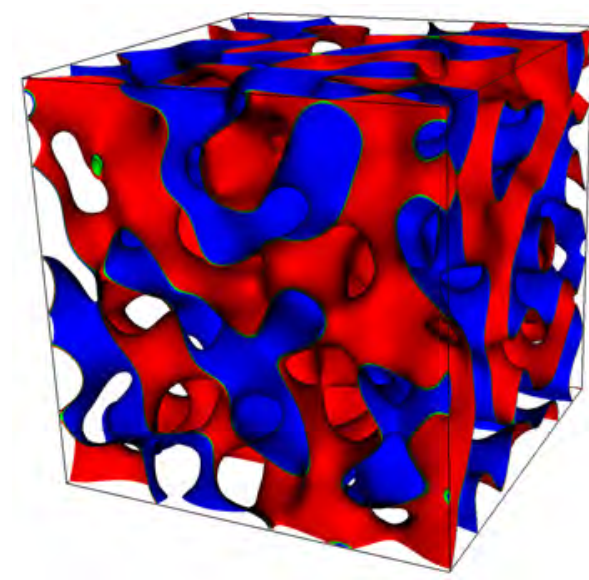

(b) $t=1$.

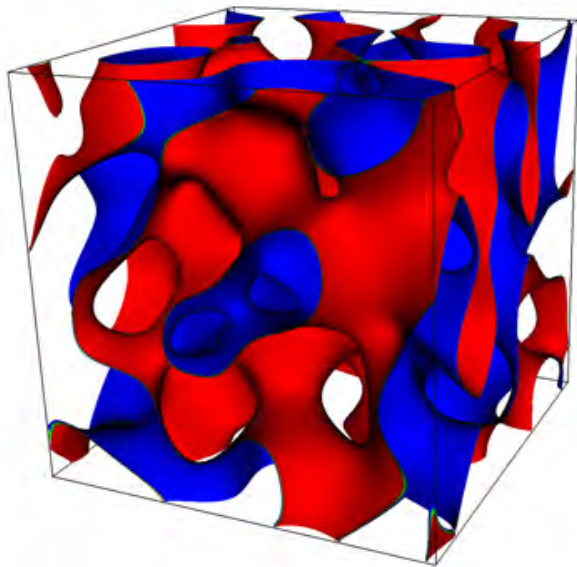

(d) $t=5$.

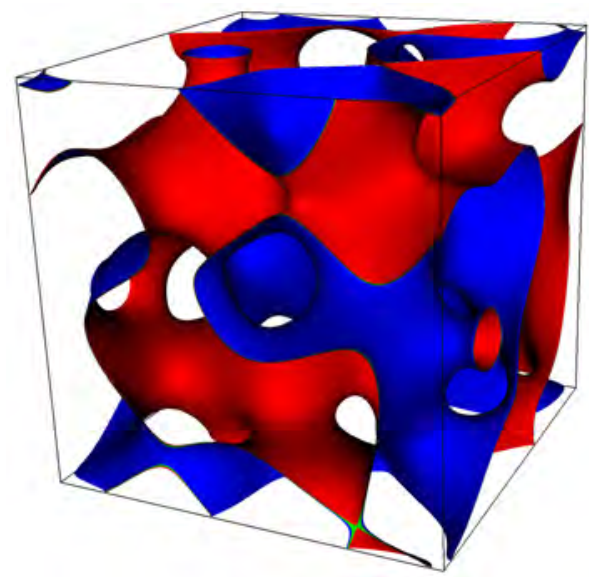

(f) $t=15$.

FIG. 4.12. The three isosurfaces of numerical solutions of the height function $\phi=-0.025,0$ and 0.025 for the No-Slope-Model of 3D coarsening dynamics using the second order scheme B-LS2 with the random initial condition and time step $\delta t=10^{-3}$. Snapshots are taken at $t=0,1,2,5,10,15$, respectively. Here we choose $\varepsilon=0.03$ 
[3] W. Chen, C. Wang, X. Wang, and S. M. Wise. A linear iteration algorithm for a second-order energy stable scheme for a thin film model without slope selection. Journal of Scientific Computing, 59(3):574-601, 2014.

[4] Z. Chen. Some new epitaxial thin film models with numerical simulations. PhD thesis, Fudan University, 2014.

[5] S. Clarke and D. D. Vvedensky. Origin of reflection high-energy electron-diffraction intensity oscillations during molecular-beam epitaxy: A computational modeling approach. Phys. Rev. Lett., 58:2235-2238, 1987.

[6] A. Diegel, C. Wang, X. Wang, and S. Wise. Convergence analysis and error estimates for a second order accurate finite element method for the cahn-hilliard-navier-stokes system. Arxiv, 2016.

[7] A. Diegel, C. Wang, and S. Wise. Stability and convergence of a second order mixed finite element method for the cahn hilliard equation. IMA Journal of Numerical Analysis, 2015.

[8] G. Ehrlich and F.G. Hudda. Atomic view of surface diffusion: Tungsten on tungsten. J. Chem. Phys., 44:1036, 1966.

[9] D. J. Eyre. Unconditionally gradient stable time marching the Cahn-Hilliard equation. In Computational and mathematical models of microstructural evolution (San Francisco, CA, 1998), volume 529 of Mater. Res. Soc. Sympos. Proc., pages 39-46. MRS, Warrendale, PA, 1998.

[10] X. Feng and A. Prohl. Error analysis of a mixed finite element method for the cahn-hilliard equation. Numerical Mathematics, 99:47-84, 2004.

[11] F. Guillén-González and G. Tierra. On linear schemes for a Cahn-Hilliard diffuse interface model. J. Comput. Phys., 234:140-171, 2013.

[12] M. F. Gyure, C. Ratsch, B. Merriman, R. E. Caflisch, S. Osher, J. J. Zinck, and D. D. Vvedensky. Level-set methods for the simulation of epitaxial phenomena. Phys. Rev. E, 58(3):69276930, 1998.

[13] D. Han, A. Brylev, X. Yang, and Z. Tan. Numerical analysis of second order, fully discrete energy stable schemes for phase field models of two phase incompressible flows. in press, DOI:10.1007/s10915-016-0279-5, J. Sci. Comput., 2016.

[14] H. C. Kang and W. H. Weinberg. Dynamic monte carlo with a proper energy barrier: Surface diffusion and twodimensional domain ordering. J. Chem. Phys., 90:2824-2830, 1989.

[15] R. V. Kohn and X. Yan. Upper bound on the coarsening rate for an epitaxial growth model. Comm. Pure Appl. Math., 56:1549-1564, 2003.

[16] J. Krug. Origins of scale invariance in growth processes. Adv. in Phys., 46:139282, 1997.

[17] B. Li and J.-G. Liu. Thin film epitaxy with or without slope selection. European J. Appl. Math., 14:713-743, 2003.

[18] D. Moldovan and L. Golubovic. Interfacial coarsening dynamics in epitaxial growth with slope selection. Phys. Rev. E, 61:6190-6214, 2000.

[19] Z. Qiao, Z. Sun, and Z. Zhang. The stability and convergence of two linearized finite difference schemes for the nonlinear epitaxial growth model. Numerical Methods for Partial Differential Equations, 28(6):1893-1915, 2012.

[20] M. Schneider, I. K. Schuller, and A. Rahman. Epitaxial growth of silicon: A moleculardynamics simulation. Phys. Rev. B, 46:1340-1343, 1987.

[21] R. L. Schwoebel. Step motion on crystal surfaces: II. J. Appl. Phys., 40:614, 1969.

$[22]$ J. Shen, C. Wang, X. Wang, and S. M. Wise. Second-order convex splitting schemes for gradient flows with ehrlichschwoebel type energy: Application to thin film epitaxy. SIAM. J. Num. Anal., 50(1):105-125, 2012.

[23] J. Shen and X. Yang. Numerical approximations of Allen-Cahn and Cahn-Hilliard equations. Disc. Conti. Dyn. Sys.-A, 28:1669-1691, 2010.

[24] J. Shen and X. Yang. A phase field model and its numerical approximation for two phase incompressible flows with different densities and viscosities. SIAM J. Sci. Comput., 32:1159-1179, 2010.

[25] G. Tierra and F. Guilln-Gonzlez. Numerical methods for solving the cahnhilliard equation and its applicability to related energy-based models. Arch. Comput. Methods Eng., 22:269289, 2015.

[26] J. Villain. Continuum models of critical growth from atomic beams with and without desorption. J. Phys. I, 19-42:13-22, 1991.

[27] C. Wang, X. Wang, and S. M. Wise. Unconditionally stable schemes for equations of thin film epitaxy. Discrete Contin. Dyn. Syst., 28(1):405-423, 2010.

[28] Y. H. Xia. A fully discrete stable discontinuous Galerkin method for the thin film epitaxy problem without slope selection. Journal of Computational Physics, 280:248-260, 2015.

[29] C. Xu and T. Tang. Stability analysis of large time-stepping methods for epitaxial growth models. Liq. Cryst., 44:1759-1779, 2006.

[30] X. Yang. Error analysis of stabilized semi-implicit method of Allen-Cahn equation. Disc. Conti. Dyn. Sys.-B, 11:1057-1070, 2009.

[31] X. Yang. Linear, first and second order and unconditionally energy stable numerical schemes for the phase field model of homopolymer blends. J. Comput. Phys., 302:509-523, 2016.

[32] X. Yang and D. Han. Linearly first- and second-order, unconditionally energy stable schemes for the phase field crystal equation. J. Comput. Phys., 330:1116-1134, 2016.

[33] X. Yang and L. Ju. Efficient linear schemes with unconditionally energy stability for the phase field elastic bending energy model. Comput. Meth. Appl. Mech. Engrg., 315:691-712, 2016.

[34] J. Zhao, Q. Wang, and X. Yang. Numerical approximations for a phase field dendritic crystal growth model based on 
the invariant energy quadratization approach,. Doi: 10.1002/nme.5372, in press, Inter. J. Num. Meth. Engr., 2016. 